\title{
Interspecies variation of larval locomotion kinematics in the genus Drosophila and its relation to habitat temperature
}

\author{
Yuji Matsuo', Akinao Nose ${ }^{1,2}$ and Hiroshi Kohsaka, ${ }^{1,3^{*}}$
}

\begin{abstract}
Background: Speed and trajectory of locomotion are the characteristic traits of individual species. Locomotion kinematics may have been shaped during evolution towards increased survival in the habitats of each species. Although kinematics of locomotion is thought to be influenced by habitats, the quantitative relation between the kinematics and environmental factors has not been fully revealed. Here, we performed comparative analyses of larval locomotion in 11 Drosophila species.

Results: We found that larval locomotion kinematics are divergent among the species. The diversity is not correlated to the body length but is correlated instead to the habitat temperature of the species. Phylogenetic analyses using Bayesian inference suggest that the evolutionary rate of the kinematics is diverse among phylogenetic tree branches.

Conclusions: The results of this study imply that the kinematics of larval locomotion has diverged in the evolutionary history of the genus Drosophila and evolved under the effects of the ambient temperature of habitats.
\end{abstract}

Keywords: Animal locomotion, Larval crawling, The genus Drosophila, Kinematics, Evolution

\section{Background}

Kinematics of animal locomotion is a critical trait enabling each species to survive in their habitats [1]. Movement patterns have been sculpted during their evolution by adaptation to their environments and could have diverged among species [2]. Comparative analyses have identified several examples of differences in the kinematics of locomotion within a group of related species, including insects [3], reptiles [4], birds [5], and primates [6]. To take an example, two gecko species inhabiting either sandy or rocky environments have been shown to

\footnotetext{
* Correspondence: kohsaka@edu.k.u-tokyo.ac.jp

${ }^{1}$ Department of Complexity Science and Engineering, Graduate School of Frontier Science, The University of Tokyo, 5-1-5 Kashiwanoha, Kashiwa, Chiba 277-8561, Japan

${ }^{3}$ School of Informatics and Engineering, The University of

Electro-Communications, 1-5-1, Chofugaoka, Chofu-shi, Tokyo 182-8585, Japan

Full list of author information is available at the end of the article
}

exhibit distinct postures [4]. Whereas the interspecies divergence in locomotion patterns can be observed in various phylogenetic branches of the animal kingdom, quantitative comparative analyses of locomotion kinematics remain limited.

Flies of the genus Drosophila have long been used as a model to study interspecific diversification and evolution [7]. One salient example of the interspecific variations in the genus Drosophila is food for larvae. Some species eat multiple kinds of foods ("generalists" including Drosophila melanogaster) while others have strong preferences in food ("specialists" including Drosophila sechellia, which has specialised to feed on Morinda fruits) [8-10]. These diverged species in the genus Drosophila offer an opportunity to perform interspecific comparisons in various animal traits including larval locomotion.

Fly larvae, or maggots, have been widely used in the study of the kinematics of locomotion [11-17]. Among

(c) The Author(s). 2021 Open Access This article is licensed under a Creative Commons Attribution 4.0 International License, which permits use, sharing, adaptation, distribution and reproduction in any medium or format, as long as you give appropriate credit to the original author(s) and the source, provide a link to the Creative Commons licence, and indicate if changes were made. The images or other third party material in this article are included in the article's Creative Commons licence, unless indicated otherwise in a credit line to the material. If material is not included in the article's Creative Commons licence and your intended use is not permitted by statutory regulation or exceeds the permitted use, you will need to obtain permission directly from the copyright holder. To view a copy of this licence, visit http://creativecommons.org/licenses/by/4.0/ The Creative Commons Public Domain Dedication waiver (http://creativecommons.org/publicdomain/zero/1.0/) applies to the data made available in this article, unless otherwise stated in a credit line to the data. 
the fly species, Drosophila melanogaster (Dmel) is one of the most examined species, especially by virtue of the availability of resources in genetics and connectomics [18-21]. Dmel larvae locomote by a sequence of forward crawling, and changes of crawling direction are achieved by bending their bodies [11]. The kinematics of larval locomotion is affected by ambient temperature [22-25]. In thermotaxis behaviour in temperature gradient environments, larvae regulate the length of crawling runs between turns and the size and direction of turns [26], and the probability of turns is also affected by ambient temperature gradients [27]. In contrast to the intensive studies on larval behaviour in Dmel, locomotion kinematics in the larvae of its sister species in the genus Drosophila remains unclear.

Here, we conducted an interspecies comparison of the kinematics of larval locomotion in the genus Drosophila. We address two questions in this study: are locomotion kinematics of larvae similar among Drosophila species? and if the kinematics are diverged, what factors are related to the diversity? To this aim, we recorded the locomotion of larvae of 11 Drosophila species and extracted kinematic parameters using the tracking software FIMTrack [28]. Clustering analysis with Jensen-Shannon divergence and statistical analyses show that two kinematics parameters (bend probability and crawling speed) differ among the Drosophila species. We found that kinematics varies with habitat temperature but not with body size. The relationship between the kinematics and minimum habitat temperature is held at two distinct ambient temperatures: $24^{\circ} \mathrm{C}$ and $32^{\circ} \mathrm{C}$. Phylogenetic analyses of these kinematics, based on Bayesian inference [29], suggest that the rate of evolution of the kinematics is diverged among phylogenetic branches. Among the eight traits we tested, the evolution of the crawling speed at $24^{\circ} \mathrm{C}$ and $32{ }^{\circ} \mathrm{C}$ was correlated. Consequently, our results suggest that the kinematics of larval locomotion in the genus Drosophila diverged in response to environmental variation in ambient temperature.

\section{Results}

Kinematic analysis of crawling and bending behaviour in Drosophila larvae

In this study, we analysed the kinematics of larval locomotion. To this aim, we recorded the locomotion of fly larvae that were crawling freely on an agarose substrate stage on a temperature-controlled plate (Fig. 1A; see the "Methods" section for details). We placed eight to ten larvae at the centre of the stage, which was kept at $24{ }^{\circ} \mathrm{C}$; illuminated them with infrared light, which was invisible to fly larvae and did not affect their behaviour; and recorded larval locomotion for $3 \mathrm{~min}$ at five frames per second (Fig. 1B). Maximum projection of the time-series images showed traces of larval locomotion as multiple curves of multiple larvae (Fig. 1C). In an example of Drosophila melanogaster (Fig. 1D), the traces showed smooth curved lines interconnected with angles where larvae exhibited turning behaviour and changed crawling direction, which was consistent with previous studies [11, 30-35]. Larvae under these conditions predominantly exhibited forward locomotion in which muscular contraction was propagating from the posterior to anterior segments. Characteristics of larval locomotion could be described by two measures: the bend angle and centroid speed [31,33]. The bend angle measures the angle of body axis bending (Fig. 1E), and the centroid speed was the speed of the position of the larval centroid (Fig. 1F). To obtain these values for each larva at each time frame, we used the object tracking software for small animals, FIMTrack [28]. The turning behaviour could be detected by the change in the angle of the body axis (Fig. 1E) and the reduction in the centroid speed (Fig. $1 F)$, as reported previously [31, 33]. Accordingly, we used the bend angle and centroid speed for the quantitative analysis of larval locomotion in this study.

\section{Classification of locomotion properties in the genus Drosophila}

For interspecies comparison of Drosophila larval locomotion, we collected 11 fly species in the genus Drosophila, whose genome sequences have been read [36, 37] and for which living individuals were available from fly stock centres (KYORIN-Fly, Fly Stocks of Kyorin University; KYOTO Stock Center (DGRC) at the Kyoto Institute of Technology). The 11 Drosophila (D., hereafter) species consisted of $D$. ananassae (Dana), D. erecta (Dere), D. mauritiana (Dmau), D. melanogaster (Dmel), D. mojavensis (Dmoj), D. persimilis (Dper), D. pseudoobscula (Dpse), D. sechellia (Dsec), D. virilis (Dvir), D. willistoni (Dwil), and D. yakuba (Dyak). Nine species (all but Dvir and Dmoj) were classified as subgenus Sophophora, and the remaining two (Dvir and Dmoj) were non-Sophophora species. We plotted the centroid speed and bend angle of freely crawling larvae of each species (Fig. 2A). In all the cases, data points accumulated around a region where the bend angle was 0 (Fig. 2A), which reflected an observation that larvae did not bend during the majority of the time (Fig. 1D). We noticed that the deviations from 0 in the bend angle axis were different among species. For example, data points in Dvir were scattered along the bend angle axis more than those in Dwil (Fig. 2A). To quantify the similarities in the distribution of the two-dimensional plots among the species, we calculated the Jensen-Shannon divergence, which measured the similarity of two probability distributions [38]. We classified the 11 species based on the similarity in the probability distribution of the crawling speed and bend angle plots by hierarchical clustering 

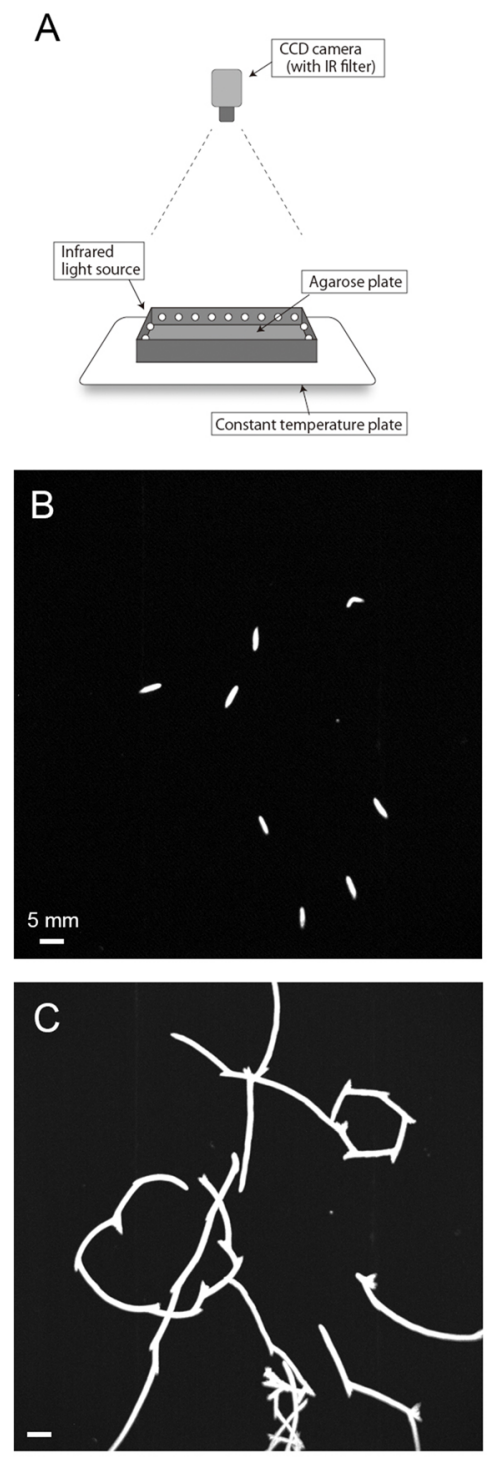

Drosophila melanogaster
D

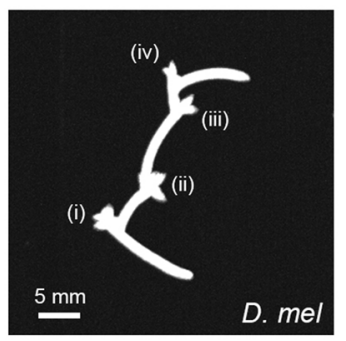

E

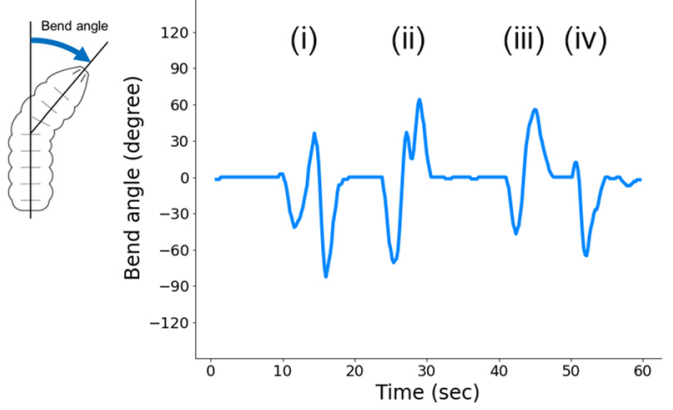

$\mathrm{F}$
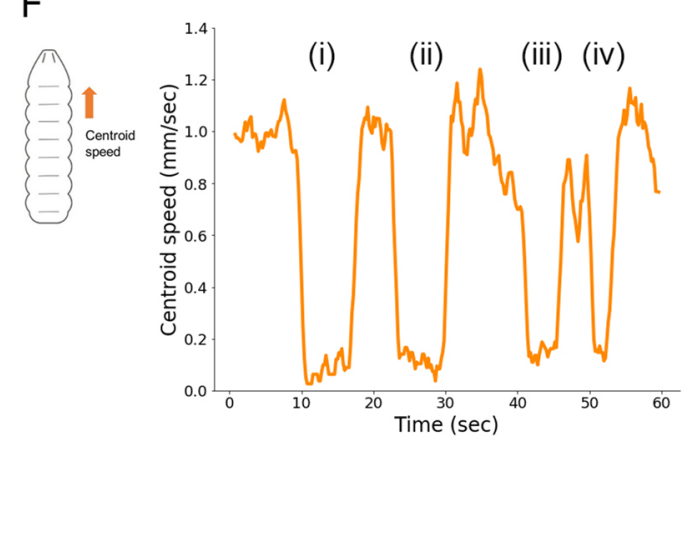

Fig. 1 Measurement of larval crawling. A Set-up of the recording of larval crawling with infrared light and temperature control plate. B An example image of multiple larvae recorded by the set-up A. C The trajectories of larval locomotion of Drosophila melanogaster recorded for 2 min. D A trajectory of single Drosophila melanogaster locomotion. Locations where the larva changes its direction were labelled (i) to (iv) (E, F). Bend angle (E) and centroid speed (F) of the larva in $\mathbf{D}$. Labels (i) to (iv) corresponded to those in $\mathbf{D}$

(Fig. 2B, C). The classification analysis showed that four species (Dmel, Dvir, Dmoj, and Dper) form a cluster. These four species showed scattered data points along the bend angle axis compared with the other seven species (Fig. 2A). This observation suggested that the kinematics of larvae in the genus Drosophila was diverse among species.

\section{Definition of the bend probability and crawling speed}

The clustering analysis suggested that the kinematics was divergent among species. To interpret the diversity in terms of locomotion behaviour, we defined two indices: the bend probability and crawling speed. The bend probability measured how often larvae bent their body laterally. We set the minimum angle of the bend as $20^{\circ}$, which was used previously [39], and labelled the larvae that bent more than this threshold angle to the right or left side as "bending" (Fig. 3A, B). This threshold allowed us to extract the difference in the bending rate among the species we examined, and we found that while Dmel larvae exhibited larger bend angles than this threshold (Fig. 3A), the bend angles in Dwil larvae were mostly less than the threshold (Fig. 3B). We defined the bend probability of every single larva as a ratio of the 


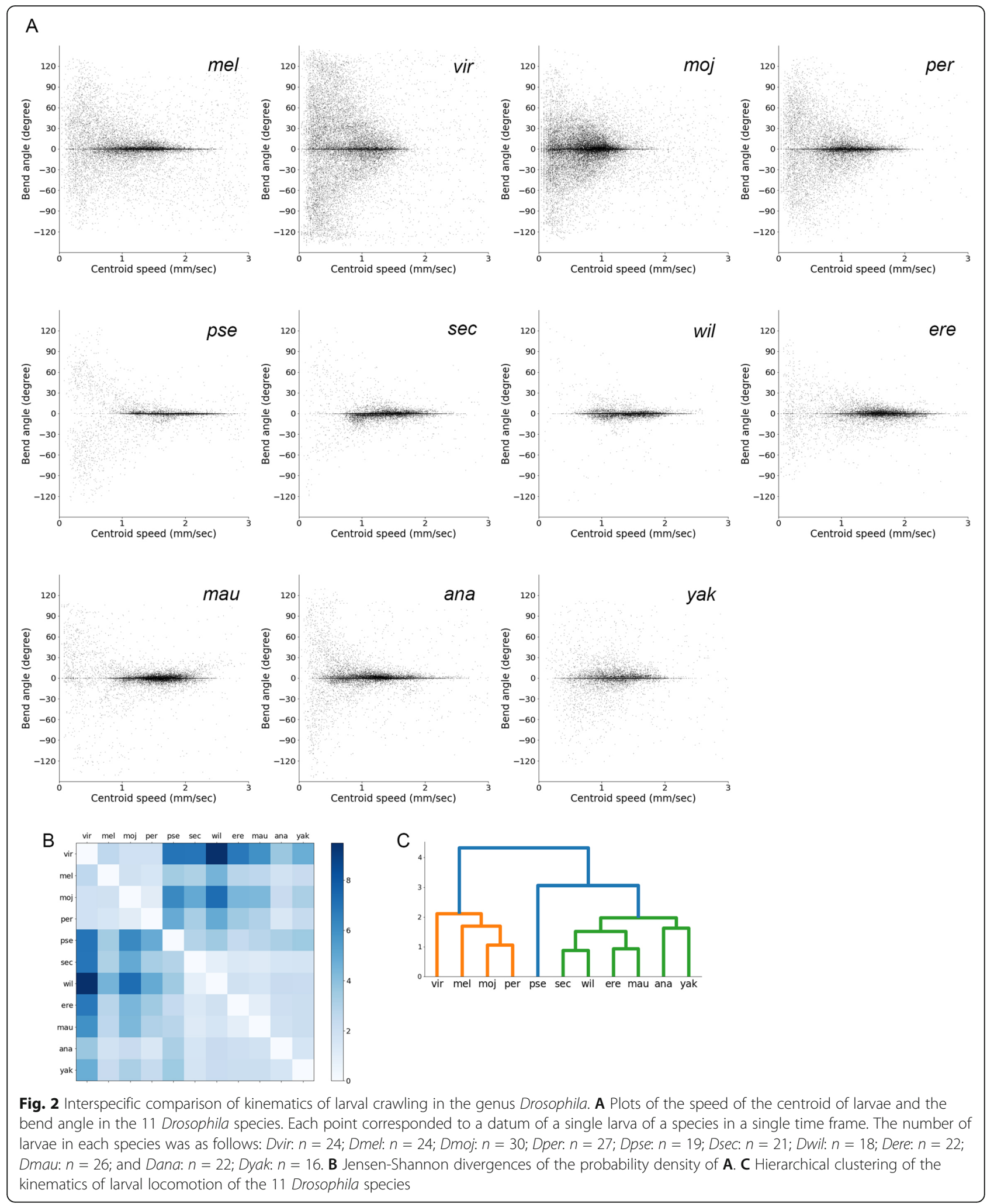

number of time frames labelled "bending" to the total frame number. To define the second index crawling speed, we labelled the larvae that bent less than the threshold as "crawling" (Fig. 3A, B). We defined the median of centroid speeds of larvae that were labelled "crawling" as the crawling speed for every single larva. 


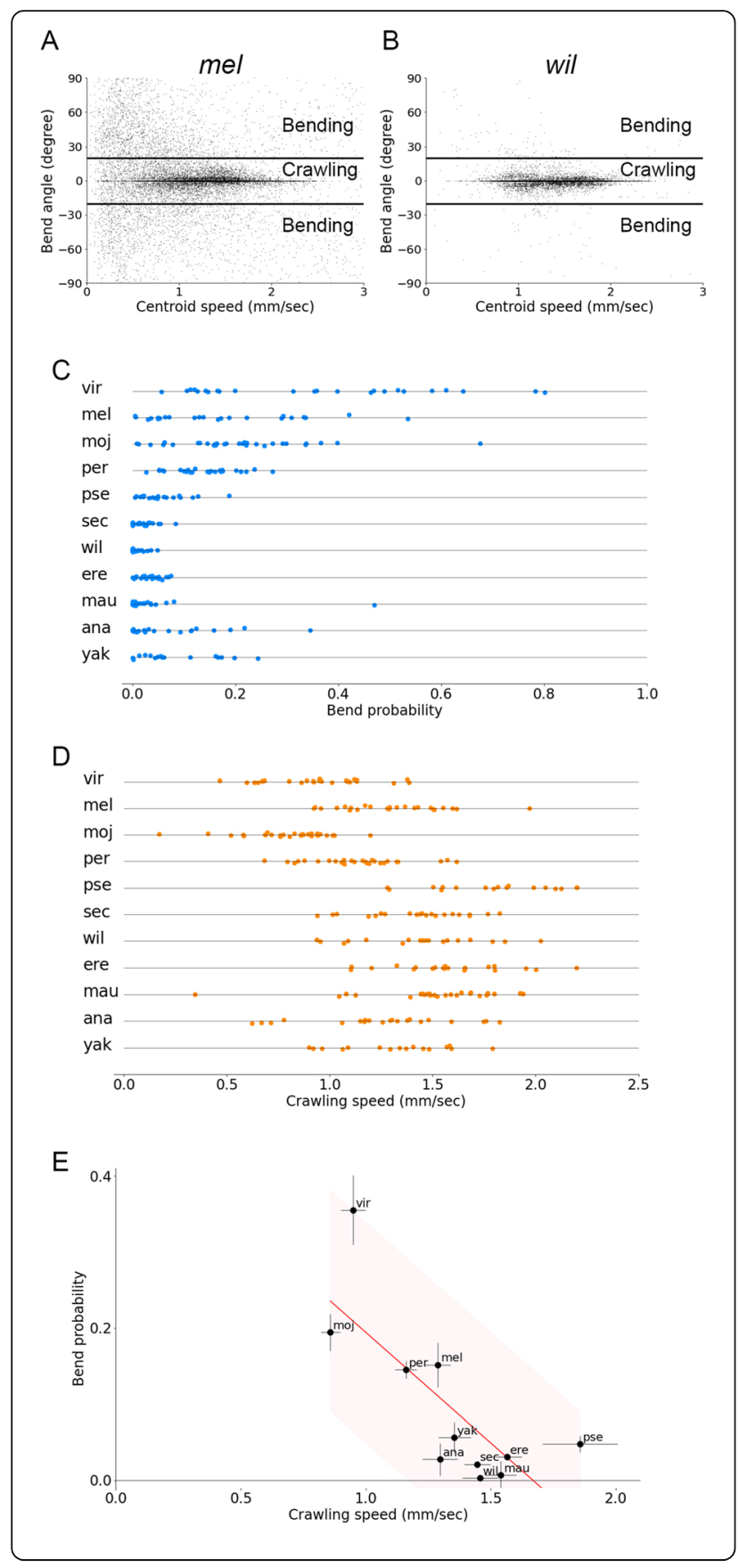

Fig. 3 Bend probability and crawling speed of larval locomotion in the 11 Drosophila species at $24^{\circ} \mathrm{C}$ and their relationship to habitat temperature of each species. A, B Plots of the speed of the centroid of larvae and the bend angle of D. melanogaster $(\mathbf{A})$ and D. willistoni (B). Each point corresponded to a datum of a single larva of a species at a single time frame. Thick horizontal lines denoted the threshold between crawling and bending. $\mathbf{C}$ Bend probability of individual larvae of each species. D Crawling speed of individual larvae of each species. Sample numbers in $\mathbf{C}$ and $\mathbf{D}$ were as follows: Dvir: $n=24 ;$ Dmel: $n=24 ;$ Dmoj: $n=30 ;$ Dper: $n=27$; Dpse: $n=19$; Dsec: $n=21$; Dwil: $n=18$; Dere: $n=22$; Dmau: $n=26$; Dana: $n=22$; and Dyak: $n=16$. E Scatter plot of bend probability at $24^{\circ} \mathrm{C}$ against crawling speed at $24^{\circ} \mathrm{C}$. The median \pm sem was shown. The red line showed the linear regression function, and the shaded area represented the $95 \%$ confidence band. The point estimate of the Pearson correlation and its $95 \%$ confidence interval was -0.76 and $[-.94,-0.30]$, respectively

By these definitions, we calculated the bend probability and crawling speed of each larva of the species.

\section{Kinematics of larval locomotion was diverse among the Drosophila species}

We plotted the bend probability of each species (Fig. $3 C)$. A statistical analysis shows that the bend probability of the species was diverse $\left(p=6.8 \times 10^{-25}\right.$, KruskalWallis test). While Dvir larvae exhibited frequent bending (bend probability $0.36 \pm 0.05, n=24$ ), Dwil larvae rarely bent (bend probability $0.003 \pm 0.004, n=18$ ) (Fig. $3 \mathrm{C}$ ). We also plotted the crawling speed of the Drosophila species (Fig. 3D). The statistical analysis showed that the speed was also diverse among the species $(p=9.3 \times$ $10^{-24}$, Kruskal-Wallis test). For example, Dwil larvae crawled faster than Dvir larvae did (crawling speed 1.46 $\pm 0.07 \mathrm{~mm} / \mathrm{s}, n=24$ in Dwil; crawling speed $0.95 \pm 0.05$ $\mathrm{mm} / \mathrm{s}, n=18$ in Dvir). These analyses indicated that the bend probability and crawling speed were differentiated in the genus Drosophila.

To capture the trends in the diversity of larval kinematics, we plotted the data in the space of the crawling speed and bend probability (Fig. 3E). The graph showed a negative correlation between them (Pearson correlation $=-0.76, p=0.0064)$. While we used $20^{\circ}$ as the threshold for defining the bend probability (Fig. 3A, B), the negative correlation between the crawling speed and bend probability was robust to the choice of the threshold (Pearson correlation $=-0.80, p=0.0030$, when the threshold was 10 degrees; Pearson correlation $=-0.71$, $p=0.014$, when the threshold was $30^{\circ}$; Additional file 1 : Supplementary Figure 1A - 1C). Furthermore, the median of absolute values of bend angle instead of the bend probability exhibited a negative correlation to the crawling speed (Pearson correlation $=-0.66, p=0.026$; Additional file 1: Supplementary Figure 1D). To sum, the crawling speed and bend probability were diverse among the Drosophila species and negatively correlated. 


\section{Comparison between intraspecies and interspecies variability}

The comparison of the 11 Drosophila species represented the natural variability in larval locomotion across them. Here, it should be noted that we used a single strain for each species in the analysis. For that, the variability appeared above could result not only from interspecies but also intraspecies diversity. Even if there was no interspecies variability, sampling data from a population with high intraspecies variation could lead to an apparent diversity across the species. To address this issue, we compared intraspecific deviation with interspecific variability. If intraspecific diversity was a dominant factor for the variability, the deviation within species should be comparable to that among species. In contrast, if interspecific diversity was a major cause, the deviation within species would be smaller than that across species. We examined intraspecies variation in two species in subgenus Sophophora (Dmel and Dana) and one nonSophophora species (Dvir) (Fig. 4). Two isofemale strains of each species were obtained from the Kyorin Stock Centre, and the larval kinematics of them were measured. To evaluate the contribution of the interspecific deviations to the total deviations, we conducted the analysis of variance (ANOVA). We found a significant interspecific difference in the bend probability $(p=0.008$, the one-way ANOVA) although the difference in the crawling speed was marginal ( $p=0.08$, the one-way ANOVA). This observation implied the existence of interspecies diversity in larval locomotion.

\section{Crawling distance was related to crawling speed and bend probability}

In the interspecies variability, there was a negative correlation between the crawling speed and bend probability (Fig. 3E). We noticed that this correlation could reflect the control of the crawling distance by a coordinated change of the crawling speed along with the bend probability. The bend probability was negatively correlated with the crawling distance since frequent bending shortened the distance larvae progress in one direction (Fig. $5 \mathrm{~A}$ ) whereas it was obvious that the crawling speed was positively correlated with the crawling distance (Fig. 5B). Accordingly, high crawling speed and low bend probability, which were a combination that appeared in the negative correlation of them (Fig. 3E), both contributed to the increase in the crawling distance. To check whether these geometrical speculations held in the locomotion of larvae, we examined the relationship between the kinematic parameters and the crawling distance (Fig. $5 \mathrm{C}, \mathrm{D})$. Consistent with the conjecture, the crawling distance was larger when either the bend probability was lower (Fig. 5C; Pearson correlation =0.78) or the crawling speed was higher (Fig. 5D; Pearson correlation $=0.67)$. These observations implied that the coordinated changes in the crawling speed and bend probability would be related to the change in the crawling distance of larvae of each species.

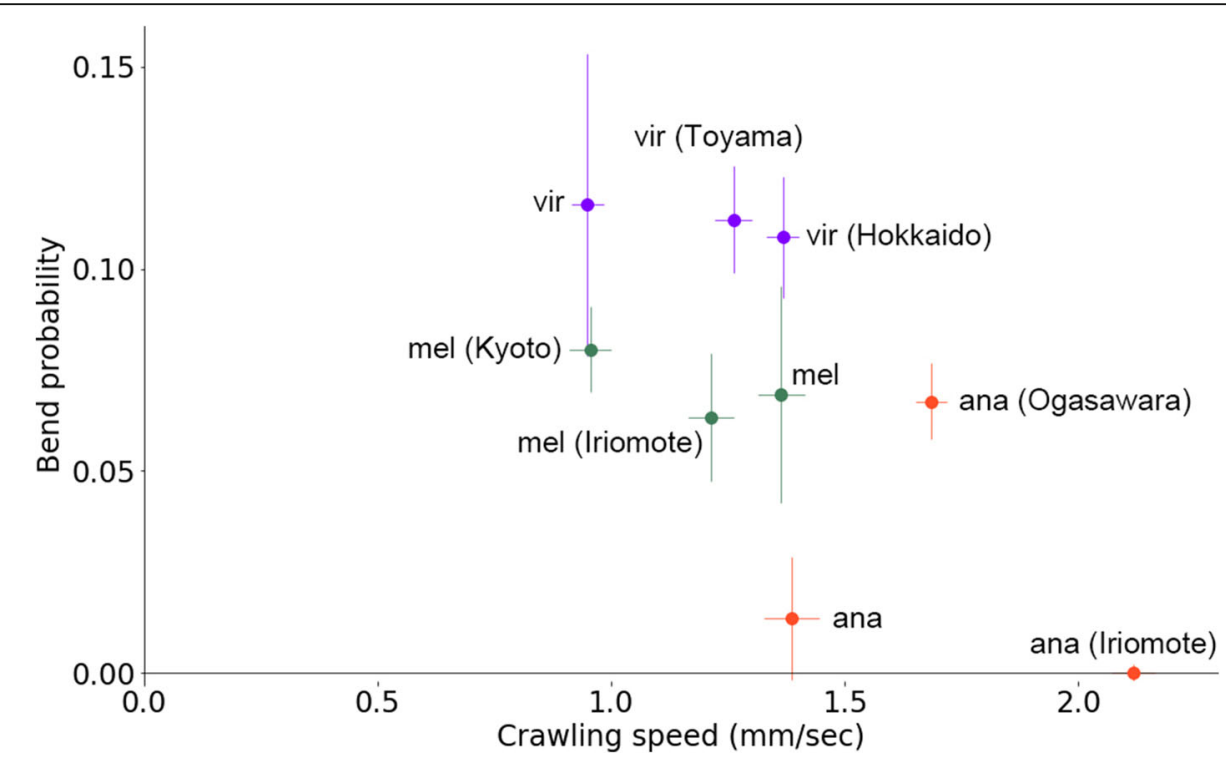

Fig. 4 Intraspecific comparison in larval locomotion. Scatter plot of bend probability at $24^{\circ} \mathrm{C}$ against crawling speed at $24^{\circ} \mathrm{C}$ of nine strains from three species. Sample numbers were as follows: Dvir: $n=44$; Dvir (Hokkaido): $n=32$; Dvir (Toyama): $n=35$; Dmel: $n=33$; Dmel (Kyoto): $n=47$; Dmel (Iriomote): $n=33$; Dana: $n=33$; Dana (Ogasawara): $n=40$; and Dana (Iriomote): $n=20$ 


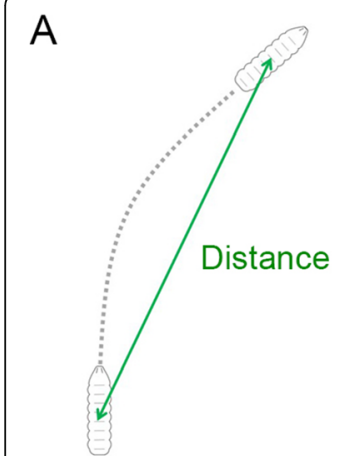

Low bend probability

B

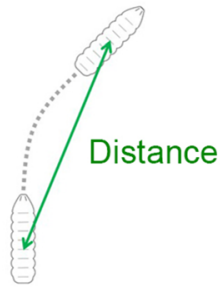

Low crawling speed

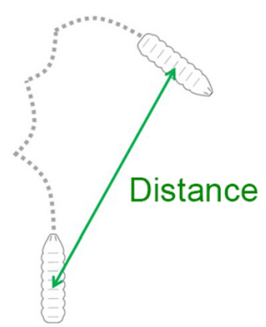

High bend probability

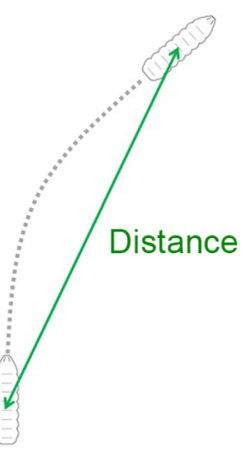

High crawling speed

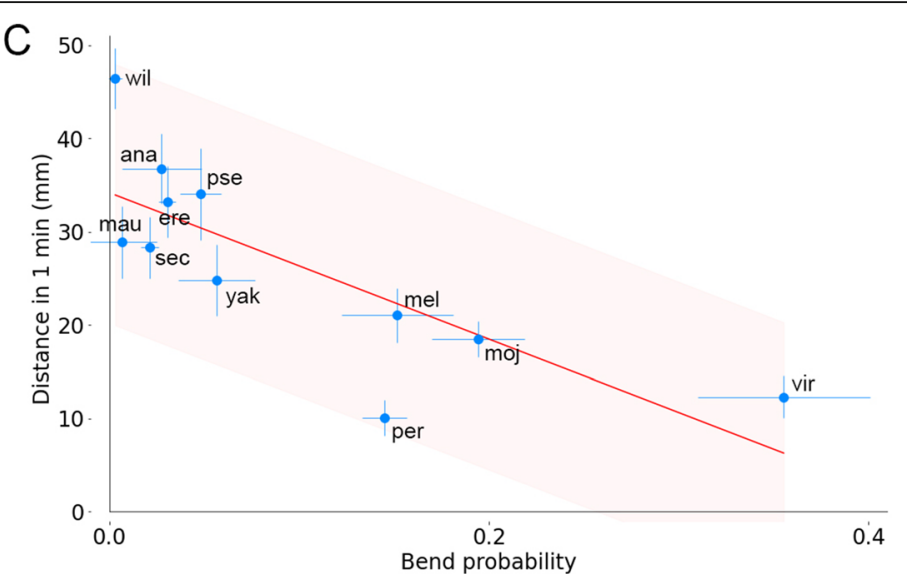

D

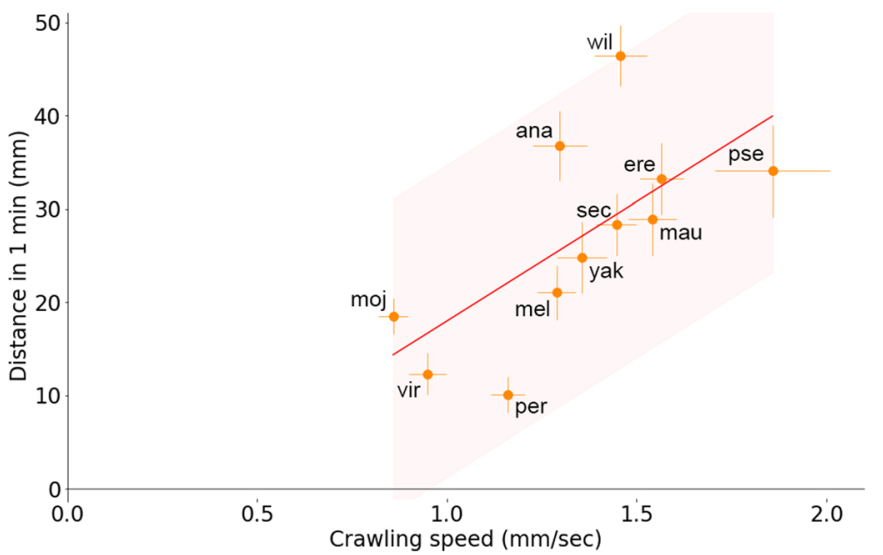

Fig. 5 Relationship between crawling distance and the two kinematic parameters in the 11 Drosophila species. A Schematics of the relationship between the bend probability and crawling distance. B Schematics of the relationship between the crawling speed and crawling distance. $\mathbf{C}$ Scatter plot of the bend probability and crawling distance in $1 \mathrm{~min}$ in the 11 species. D Scatter plot of the crawling speed and crawling distance in $1 \mathrm{~min}$ in the 11 species. The source locomotion data were the same as in Fig. 3C, D. In $\mathbf{C}$ and $\mathbf{D}$, the red lines showed the linear regression functions, and the shaded areas represented the 95\% confidence bands. The point estimates of the Pearson correlation and their $95 \%$ confidence intervals were -0.78 and $[-0.94,-0.34]$ in $\mathbf{C}$ and 0.67 and $[0.11,0.91]$ in $\mathbf{D}$, respectively

\section{No relationship between the kinematics of larval locomotion and the body length nor phylogenetic relationship}

Next, we tried to find factors that relate to the diversification of larval kinematics among species. Among the factors in the morphological differences and the ecological diversity that might be involved in the kinematics diversity [3], we examined the body length of larvae as a morphological factor and habitat temperature as an ecological factor. A previous study reported an allometric relationship between the body size and the crawling speed in Diptera larvae [13]. The authors analysed larvae in the order Diptera; the average size of which spans from $3.7 \mathrm{~mm}$ (Dmel) to $15.9 \mathrm{~mm}$ (Sarcophaga bullata). We tested whether the relationship between the body length and the crawling speed also held within the genus Drosophila, a subgroup of the order Diptera. The length of the Drosophila larvae we used spanned from $3.49 \pm$
$0.05 \mathrm{~mm}$ (Dyak) to $5.68 \pm 0.16 \mathrm{~mm}$ (Dpse) (Additional file 1: Supplementary Figure 2A). We found no significant relationship between the body length and crawling speed (Additional file 1: Supplementary Figure 2B; Pearson correlation $=0.04, p=0.91$ ) and between the body length and the bend probability (Additional file 1: Supplementary Figure 2C; Pearson correlation $=0.55, p=$ 0.077). These data suggested that larval length was not a significant factor for larval kinematics variation within the genus Drosophila.

The phylogenetic relationship could also be a factor that affects the kinematics. To test this issue, we focused on two species groups: the obscura species group and the replete species group. Dper and Dpse belonged to the obscura species group (see Fig. 11A). The kinematics of these two sister species were separated in the distribution of the crawling speed and bend probability (Fig. 3E). Similarly, two species of the replete species group, Dvir 
and Dmor, exhibited distinct kinematics (Fig. 3E). These observations suggested that the phylogenetic relationship was not a major factor in the divergence of the kinematics.

\section{Relationship between the kinematics of larval locomotion and habitat temperature of the Drosophila species}

Habitat temperature was one of the critical factors influencing species traits [40]. To test the possible roles of habitat temperature in the evolution of Drosophila larval locomotion, we examined the relationship between habitat temperatures and the locomotion kinematics in the genus Drosophila. The habitat regions of the 11 species were obtained from the literature $[7,41,42]$, and the climate temperature data were obtained from a global climate dataset, WORLDCLIM [43] (Fig. 6A, Additional file 1: Supplementary Figure 3). We examined the relation between larval kinematics (bend probability or crawling speed) and indices of habitat temperatures (Fig. $6 \mathrm{~B}, \mathrm{C}$ ). We used the mode (the most frequent value) of three indices of habitat temperatures: average, maximum, and minimum temperature. In the minimum habitat temperature data of Dvir and Dpse, we noticed that the mode temperatures were below $0^{\circ} \mathrm{C}$, which cannot be a representative habitat temperature for them (Additional file 1: Supplementary Figure 3). Accordingly, for a milder and more representative index, we used the warmest peaks in the minimum habitat temperature histogram ("Tmin" in Additional file 1: Supplementary Figure 3). Considering the uneven distribution of flies within the region demarcated in Additional file 1: Supplementary Figure 3, the temperature of the warmest peak in the minimum habitat temperature might represent the coldest temperature the majority of the population of each species experiences over many years.

We found that the bend probability and crawling speed were both correlated with average habitat temperature Tave (Fig. 6B, E. Pearson correlation: bend probability vs Tave $=-0.73$, crawling speed vs Tave $=$ 0.66). We further analysed whether the maximum and minimum temperature contribute to the correlation. Maximum habitat temperature, Tmax, showed no obvious correlation to the kinematics (Fig. 6C, F. Pearson correlation: bend probability vs $T \max =0.37$, crawling speed vs Tmax $=-0.31$ ). In contrast, the minimum habitat temperature, Tmin, exhibited a stronger correlation than Tmax (Fig. 6D, G. Pearson correlation: bend probability vs Tmin $=-0.81$, crawling speed vs Tmin $=$ 0.74). Even after omitting extreme values (Dvir in the bend probability data and Dpse in the crawling speed data), the correlation remained high (Pearson correlation: the bend probability vs Tmin (without Dvir) = 0.69 , the crawling speed vs Tmin (without $D p s e)=0.78$ ). Furthermore, even considering multiple comparisons (four factors: larval length, Tave, Tmax, and Tmin), the correlations between the kinematic parameters and Tmin were statistically significant $(p=0.039$ in crawling speed vs Tmin, $p=0.0010$ in bend probability vs Tmin; Bonferroni correction).

The range (or variability) of habitat temperature could also be a critical factor to determine larval kinematics because species that inhabits in highly variable temperature area would be insensitive to the change in the ambient temperature whereas those breeding in a narrow range of temperature would be sensitive to the small shift in the ambient temperature. To test this point, we examined the relationship between the larval kinematics and the range of habitat temperature and the difference between the maximum and minimum temperature in their habitat area (Additional file 1: Supplementary Figure 4). The range of habitat temperature was correlated with both the bend probability (Pearson correlation $=0.65, p=0.030$ ) and the crawling speed (Pearson correlation $=-0.69, p=0.0019)$. Consequently, the range of habitat temperature could affect the larval kinematics. In the following analysis, we focused on the minimum temperature Tmin since it showed the most evident correlation to the larval kinematics.

It should be noted that the strains we used have been kept at the housed stocks at 23 or $20^{\circ} \mathrm{C}$ (see the "Methods" section), which might affect the innate behaviour in wild-type strains. However, a comparison between the time period after speciation (at least million years $\sim 10^{7}$ generations $[44,45]$ ) and that in laboratories (100 years $\sim 10^{3.5}$ generations) indicated that the duration in laboratories occupied as small as $0.03 \%$ of the time for the evolution of these Drosophila strains. In addition, the rearing temperatures in the stock centres were within the range of habitat temperature of each species (Additional file 1: Supplementary Figure 3). These rearing conditions implied that the strains we collected from the stock centre should possess innate behaviour that was evolved in their habitat.

To sum, larvae of species inhabiting moderate environments (where $\operatorname{Tmin}$ is 15 to $25^{\circ} \mathrm{C}$ ) showed low bend probability and fast crawling, or long crawling distance, whereas those inhabiting cold environments (where Tmin is 0 to $10^{\circ} \mathrm{C}$ ) exhibited frequent bending and slow crawling, or short crawling distance. Especially, species that had lower Tmin than the ambient temperature in this assay $\left(24^{\circ} \mathrm{C}\right)$ showed shorter crawling distance, which implied that excess heat stimuli to these species should reduce their crawling distance.

In the analysis above, we estimated the indices of habitat temperature from the temperature data in the entire potential habitat of each species because the original habitat of each strain was unclear. To further check the correlation between the larval kinematics and habitat 


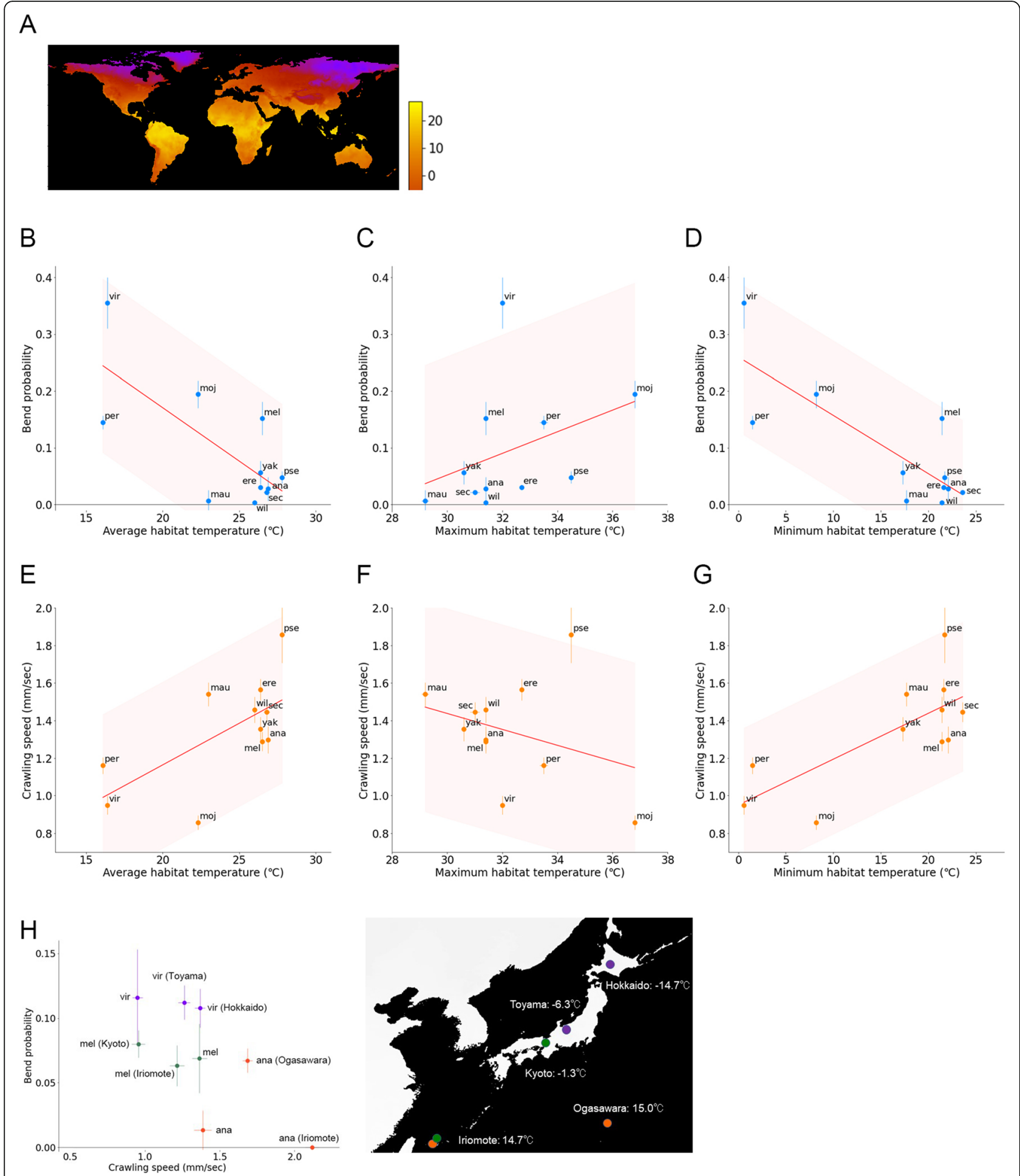

Fig. 6 Relationship between the kinematics of lanval locomotion and habitat temperature of the Drosophila species. A A world map of minimum habitat temperature. B-D Scatter plot of the bend probability of the 11 species at $24^{\circ} \mathrm{C}$ against the average (B), maximum (C), and minimum (D) habitat temperatures. $\mathbf{E}-$ $\mathbf{G}$ Scatter plot of the crawling speed of the 11 species at $24^{\circ} \mathrm{C}$ against the average $(\mathbf{E})$, maximum $(\mathbf{F})$, and minimum $(\mathbf{G})$ habitat temperatures. In $\mathbf{B}-\mathbf{G}$, the median \pm sem was shown, and the source locomotion data are the same as in Fig. 3C, D. The red lines showed the linear regression functions, and the shaded areas represented the $95 \%$ confidence bands. The point estimates of the Pearson correlation and their $95 \%$ confidence intervals were -0.73 and $[-0.93,-0.23]$ in $\mathbf{B}, 0.37$ and $[-0.30,0.79]$ in $\mathbf{C}$, and -0.81 and $[-0.95,-0.41]$ in $\mathbf{D}, 0.66$ and $[0.09,0.90]$ in $\mathbf{E},-0.31$ and $[-0.77,0.36]$ in $\mathbf{F}$, and 0.74 and $[0.25,0.93]$ in $\mathbf{G}$. $\mathbf{H}$ Left: a scatter plot of intraspecific comparison (the same as Fig. 4); right: a map of Japan representing the minimum habitat temperatures of the six strains shown in the left panel 
temperature, we examined the relationship between the kinematics and habitat temperature among the strains of which habitat was recorded (Figs. 4 and $6 \mathrm{H}$ ). We used two Dmel strains (collected at Kyoto and Iriomote in Japan), two Dvir strains (collected at Hokkaido and Toyama in Japan), and two Dana strains (collected at Ogasawara and Iriomote in Japan). The habitat temperature at these locations for collecting flies was diverse (Fig. $6 \mathrm{H}$, right). Intriguingly, the strains originated from the north part of Japan, where the minimum habitat temperature was low, exhibited slower crawling and high bend probability whereas those from the south part of Japan, where the minimum habitat temperature was high, showed the opposite trend (Fig. 6H, left), which was consistent with the observation based on the temperature of the worldwide statistics (Fig. 6D, G). Consequently, these observations implied that habitat temperature should be one of the leading factors in sculpting the kinematics of larval locomotion in the genus Drosophila.

\section{Correlation between bend probability and habitat temperature among Drosophila species at high ambient temperature}

So far, we analysed larval locomotion at $24{ }^{\circ} \mathrm{C}$, which was close to the rearing temperature (see the "Methods" section). Larvae of Dmel were known to crawl faster at $32^{\circ} \mathrm{C}$ than at $25^{\circ} \mathrm{C}$ [25]. So, we next examined whether the relation between the kinematics indices and habitat temperature held or not, and how the kinematics indices were changed, at a higher ambient temperature. We performed the same set of measurements and analysis of larval locomotion at $32^{\circ} \mathrm{C}$ (Figs. 7 and 8). The interspecies comparison showed that the bend probabilities at $32{ }^{\circ} \mathrm{C}$ were correlated with minimum habitat temperature (Fig. 7B; Pearson correlation $=-0.85, p=$ 0.0009). To examine the effects of ambient temperature on the bend probability in detail, we compared the differences of bend probability at $24{ }^{\circ} \mathrm{C}$ and $32{ }^{\circ} \mathrm{C}$ among the species (Fig. 7C). We found no significant relationship between the change in bend probability and the minimum habitat temperature (Fig. 7C; Pearson correlation $=0.40, p=0.22$ ). Seven species showed no significant changes in the bend probability at $32^{\circ} \mathrm{C}$ (MannWhitney $U$ test of Dmoj, Dper, Dpse, Dsec, Dmau, Dana, and Dyak in Fig. 7A-D), while Dvir and Dmel showed a decrease in the bend probability at $32{ }^{\circ} \mathrm{C}$, and Dwil and Dere exhibited an increase (Mann-Whitney $U$ test in Fig. $7 \mathrm{~A}-\mathrm{C}, \mathrm{E}, \mathrm{F})$. Accordingly, we concluded that while the shift of the bend probability between the distinct temperatures was diverse among the Drosophila species, the overall trend between the bend probability in relation to habitat temperature held at the higher ambient temperature of $32^{\circ} \mathrm{C}$.

\section{Correlation between crawling speed and habitat temperature among Drosophila species at high temperature}

Next, we analysed the crawling speed at $32{ }^{\circ} \mathrm{C}$ (Fig. 8A). Similar to the bend probability, we found that the crawling speed at $32^{\circ} \mathrm{C}$ was correlated with the minimum habitat temperature among the species, as it was at $24{ }^{\circ} \mathrm{C}$ (Fig. 8B; Pearson correlation $=0.67, p=0.024$ ). To examine the effects of ambient temperature on the crawling speed in detail, we compared the difference in the crawling speed at 24 to $32^{\circ} \mathrm{C}$ among the species (Fig. $8 \mathrm{C})$. In contrast to the case in the bend probability, shifts in the crawling speed among the species were common: ten species (all the species but Dper) showed a significant increase in crawling speed at $32{ }^{\circ} \mathrm{C}$ (Mann-Whitney $U$ test in Fig. 8A). Dper also exhibited an increase in the crawling speed, but it was not statistically significant (Mann-Whitney $U$ test in Fig. 8A). Accordingly, at $32^{\circ} \mathrm{C}$, larvae of all the species we tested increased their speed of crawling, which might reflect a general demand to avoid malfunctions in metabolic reactions and dehydration of the body. We also tested the relationship between the change in the speed at $32{ }^{\circ} \mathrm{C}$ and the minimum habitat temperature for each species (Fig. 8C). We found no significant correlation between the speed change and the habitat temperature (Fig. 8C; Pearson correlation $=0.07$, $p=0.84$ ), which also implied that the speeding up at high temperature was a general requirement among the species for larval survival. Accordingly, whereas the details of shifts were different (diverse shift in the bend probability and common shift in the crawling speed among the species), the overall relationship between the kinematic indices and minimum habitat temperature was also held at the higher ambient temperature of $32{ }^{\circ} \mathrm{C}$.

\section{A kinematic trend of larval locomotion in the genus Drosophila}

To see any gross trend in the shift of larval kinematics in the different species at distinct ambient temperatures, we plotted the kinematics of individual larvae of all the species (Fig. 9). At the ambient temperature of $24^{\circ} \mathrm{C}$, a trend of kinematics was observed in which the data points were distributed from low crawling speed/high bend probability (the top-left corner in the plot) to high crawling speed/low bend probability (the bottom-right corner in the plot) as the minimum habitat temperature increased (Fig. 9A). Intriguingly, the kinematics indices between distinct species were not segregated but rather overlapped. This continuum property can also be observed in the kinematics data at the ambient temperature of $32{ }^{\circ} \mathrm{C}$ (Fig. 9B). According to the modern phylogenetic concept [7], the 11 genus Drosophila species in our study consisted of 


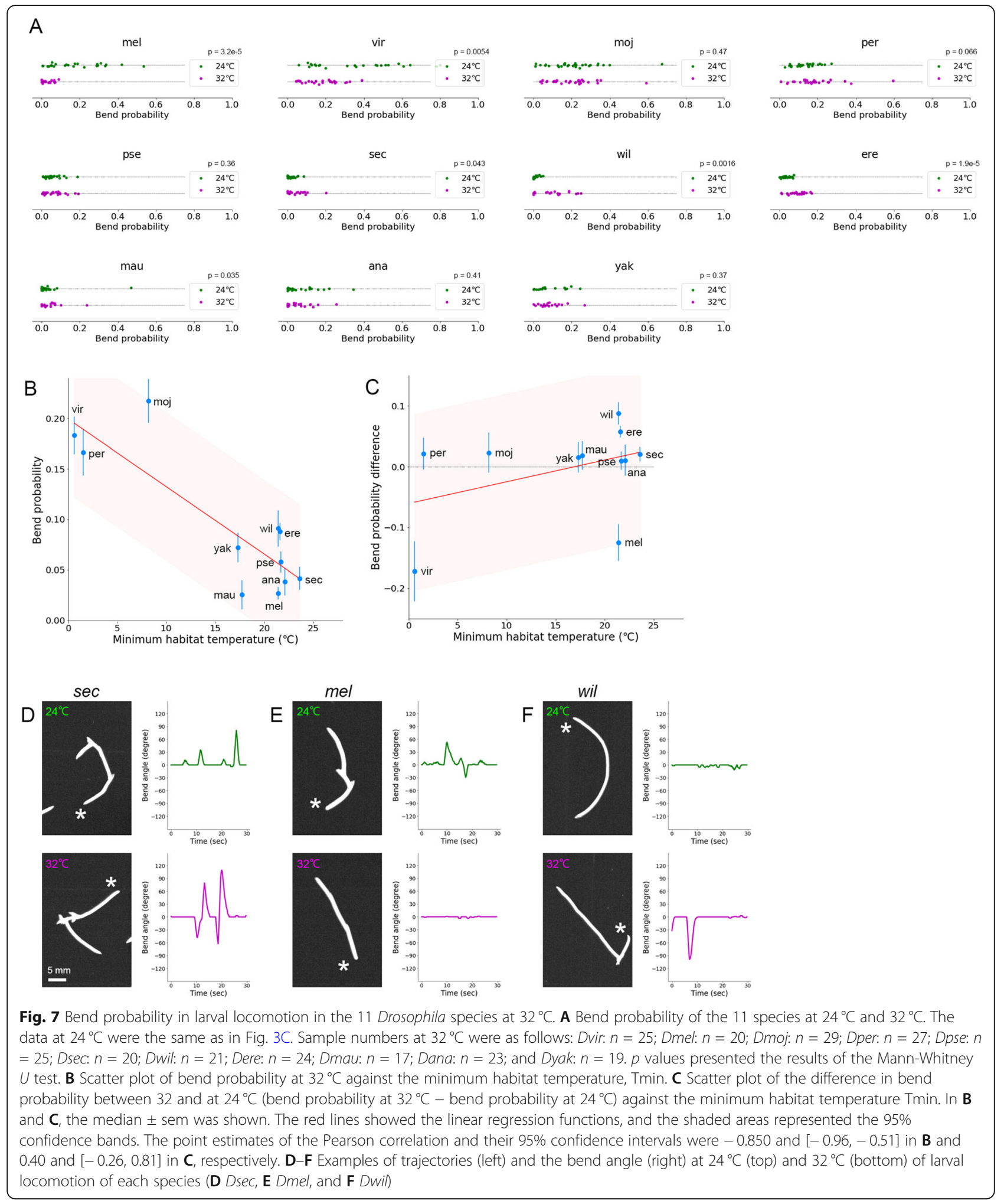

nine subgenus Sophophora species (all but Dvir and Dmoj) and two non-Sophophora species (Dvir and $D m o j)$. In our plots of kinematics, species in the nonSophophora (Dvir and Dmoj) were located at the left side in the continuum (Fig. 9). This observation implied that the kinematics indices of larvae in Sophophora species in the genus Drosophila took values in this continuum and kinematics indices in non- 


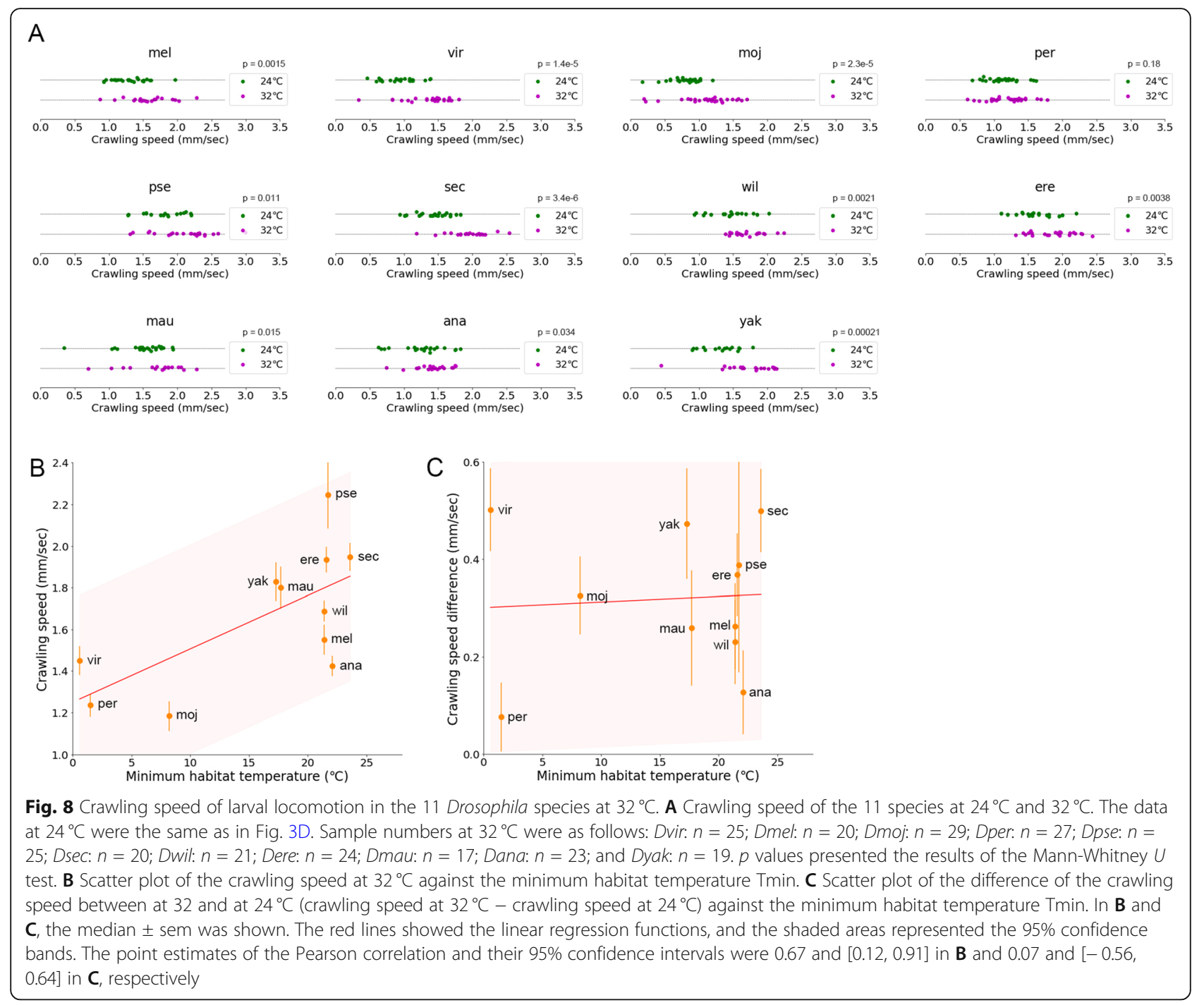

Sophophora species in the genus Drosophila diverged along this continuum by adaptation to habitat temperature during evolution.

\section{No correlation between crawling speed and habitat temperature among Drosophila species at an extreme ambient temperature of $40^{\circ} \mathrm{C}$}

Temperatures of $24{ }^{\circ} \mathrm{C}$ and $32{ }^{\circ} \mathrm{C}$ were within the range of natural habitat temperatures of most of the species we tested (Additional file 1: Supplementary Figure 3). We next examined larval crawling at an extreme temperature and investigated the relation between the kinematics indices and minimum habitat temperature. Too high temperature could be noxious for many animals, including Drosophila larvae. When D. melanogaster larvae were stimulated with a probe heated to $42^{\circ} \mathrm{C}$, they exhibited a stereotyped rolling motion as an escape behaviour [46-48]. At below $40^{\circ} \mathrm{C}$, on the other hand, larvae did not exhibit rolling behaviour [47], which allowed us to study crawling kinematics in this seminoxious extreme environment. We recorded larval locomotion of the Drosophila species at the ambient temperature of $40^{\circ} \mathrm{C}$, which corresponded to the highest edge of maximum habitat temperature histograms for many of the species (Additional file 1: Supplementary Figure 3). We measured the bend probability and crawling speed of the 11 Drosophila species (Additional file 1: Supplementary Figures 5 and 6). At the ambient temperature of $40^{\circ} \mathrm{C}$, neither the bend probability nor crawling speed was correlated with minimum habitat temperature (Fig. 10A, B; Pearson correlation: bend probability at $40^{\circ} \mathrm{C}$ vs Tmin $=0.17, p=0.61$; crawling speed at $40^{\circ} \mathrm{C}$ vs Tmin $=-0.17, p=0.61$ ). Since $40^{\circ} \mathrm{C}$ was close to the maximum habitat temperature rather than the minimum habitat temperature, we analysed the relation between the kinematics indices and maximum habitat temperature instead of the minimum habitat temperature. However, neither the bend probability nor 

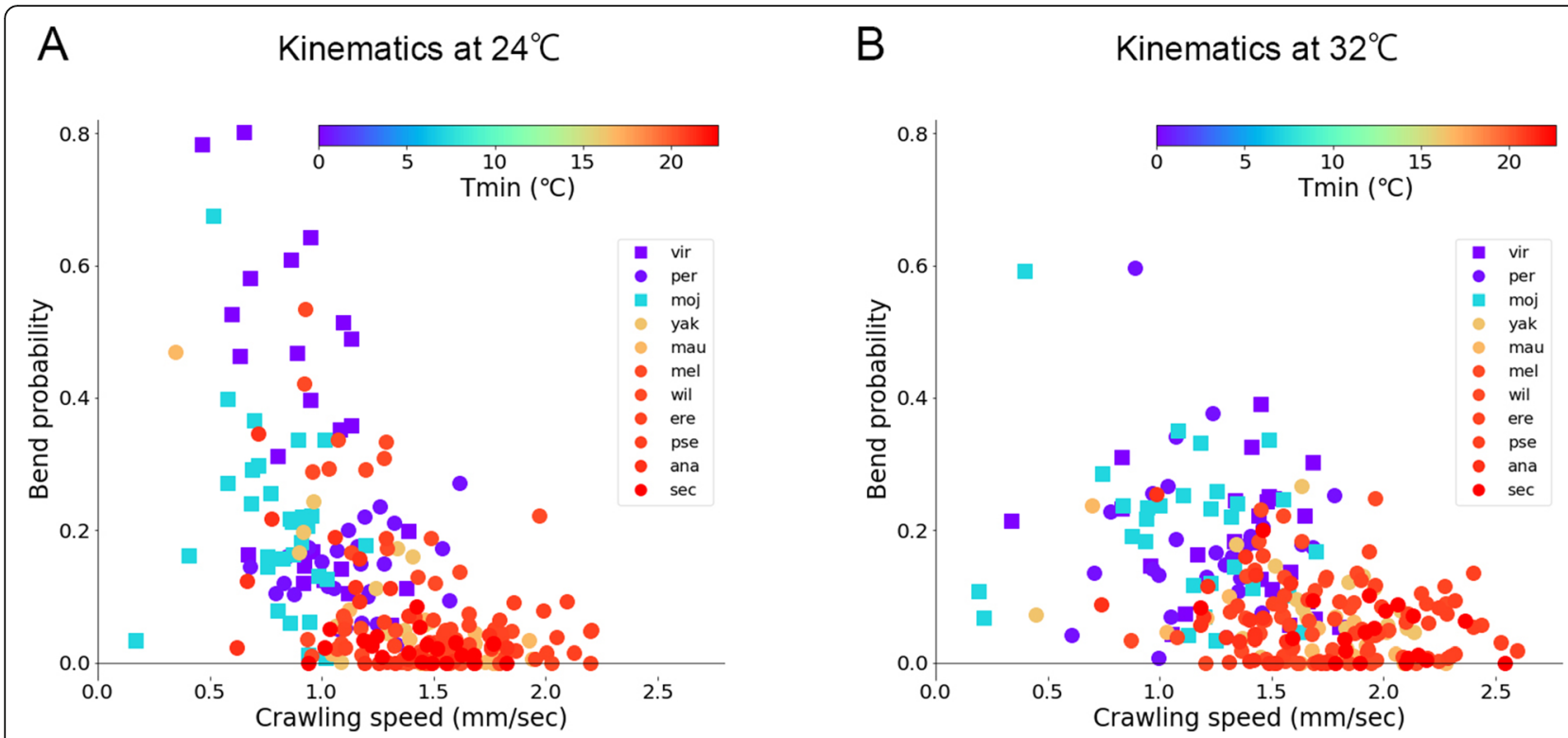

Fig. 9 Scatter plot of the kinematics in the 11 species at $24^{\circ} \mathrm{C}$ and $32^{\circ} \mathrm{C}$. A, B Two-dimensional plots of the crawling speed and bend probability. Each point corresponded to the data of a single larva of the species. The colour of markers denoted minimum habitat temperature (Tmin) as shown in the colour bar. Circles denoted Sophophora species, and squares denoted non-Sophophora species. The source locomotion data were the same as Figs. 3,7 , and 8. A $24^{\circ} \mathrm{C}$. B $32^{\circ} \mathrm{C}$

crawling speed was correlated with maximum habitat temperature (Pearson correlation: bend probability at $40^{\circ} \mathrm{C}$ vs maximum habitat temperature $=-0.13, p=$ 0.71 ; crawling speed vs maximum habitat temperature $=$ $-0.10, p=0.77)$. Accordingly, the relation between the kinematic indices and habitat temperature did not hold at the extreme ambient temperature of $40^{\circ} \mathrm{C}$. This observation suggested that the influence of habitat temperature on the evolution of the locomotion kinematics was restricted within a specific range of ambient temperatures. To examine the effects of the extreme ambient temperature on the kinematics indices in detail, we examined the shift in the bend probability at the distinct temperatures of $40^{\circ} \mathrm{C}$ and $32^{\circ} \mathrm{C}$ among the species (Additional file 1: Supplementary Figure 5). In seven species, bend probability increased at $40^{\circ} \mathrm{C}$. Three species that inhabited moderate temperature areas (Dvir, Dper, and Dmoj) and one species that lived on an isolated island (Dsec) showed little change in the bend probability, which might reflect a distinct strategy in evolution to cope with the semi-noxious environment. We also examined the shift in the crawling speed at the distinct temperatures of $40{ }^{\circ} \mathrm{C}$ and $32{ }^{\circ} \mathrm{C}$ among the species (Additional file 1: Supplementary Figure 5). We found the crawling speed was reduced at $40^{\circ} \mathrm{C}$ in all the species (Additional file 1: Supplementary Figure 6), which might be a common adaptation of the Drosophila larval locomotion at the extreme ambient temperature and/or due to an abnormal physiological reaction at the semi-noxious temperature. The similar tendency, the increase in the bend probability and the decrease in the crawling speed at $40^{\circ} \mathrm{C}$, was also observed when compared with the kinematics at $24^{\circ} \mathrm{C}$ (Fig. 10C, D). Intriguingly, in some species, backward crawling, which seldom occurs at $32^{\circ} \mathrm{C}$, could be observed at $40^{\circ} \mathrm{C}$ (Fig. $10 \mathrm{E})$. Consequently, these observations showed that at the extreme ambient temperature of $40^{\circ} \mathrm{C}$, the relation between the kinematics indices and habitat temperature did not hold and larvae exhibited common (increase in the crawling speed) and diverse (changes in the bend probability and generation of backward crawling) shifts in the locomotion kinematics among the Drosophila species.

\section{Phylogenetic analyses of interspecies differences in kinematics}

Finally, to obtain a hint on how the kinematics parameters diversified across the species developed during the evolutionary history of the genus Drosophila, we conducted Bayesian phylogenetic analyses using RevBayes v. 1.0.7 [29, 49]. At first, we inferred phylogenetic trees of the 11 Drosophila species based on eight nuclear genes used previously [50] (see the "Methods" section for details). Then, by using our kinematics dataset, we estimated the following three factors in the phylogenetic tree by Bayesian inference: the rate of evolution at each branch of the phylogenetic trees, the relative rates of evolution among the kinematic parameters, and the correlation between the kinematic parameter evolutions [51]. To perform the inference, we constructed a data 


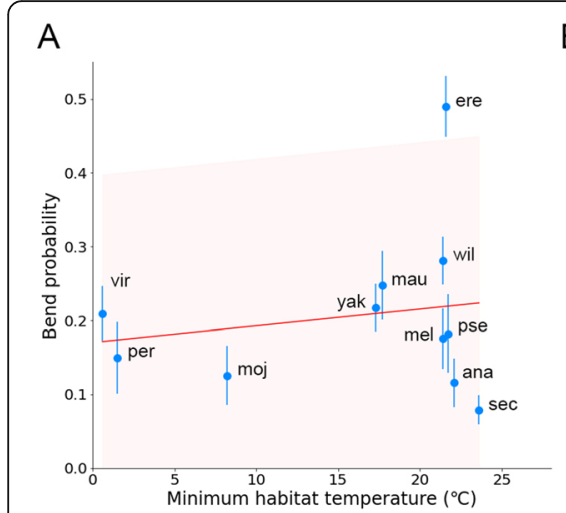

B

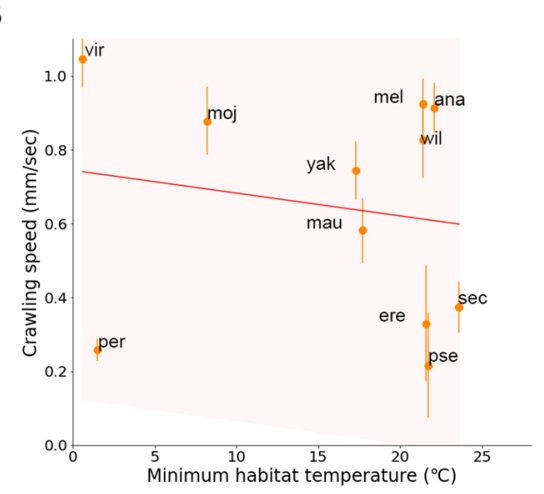

D
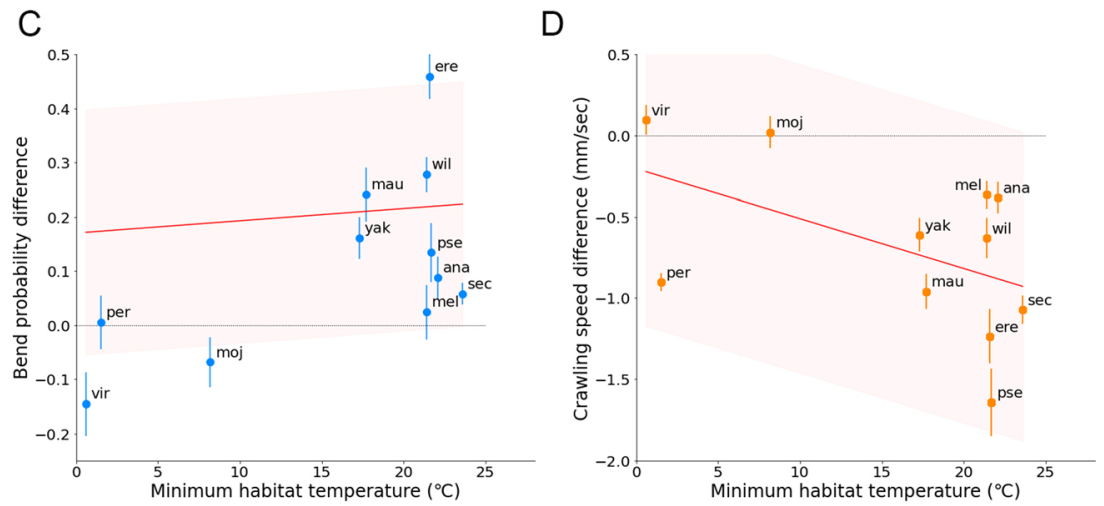

$\mathrm{E}$

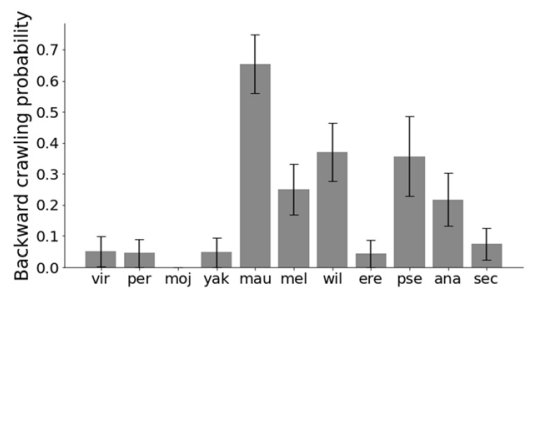

Fig. 10 Larval behaviour in the 11 species at $40^{\circ} \mathrm{C}$. A Scatter plot of the bend probability at $40^{\circ} \mathrm{C}$ against the minimum habitat temperature Tmin. B Scatter plot of the crawling speed at $40^{\circ} \mathrm{C}$ against the minimum habitat temperature Tmin. $\mathbf{C}$ Scatter plot of the difference of the bend probability between at 40 and at $24^{\circ} \mathrm{C}$ (bend probability at $40^{\circ} \mathrm{C}$ - bend probability at $24^{\circ} \mathrm{C}$ ) against the minimum habitat temperature Tmin. $\mathbf{D}$ Scatter plot of the difference of the crawling speed between 40 and at $24^{\circ} \mathrm{C}$ (crawling speed at $40^{\circ} \mathrm{C}-$ crawling speed at $24^{\circ} \mathrm{C}$ ) against the minimum habitat temperature Tmin. The median \pm sem was shown in A-D. Sample numbers for data at $40^{\circ} \mathrm{C}$ were as follows: Dvir: $n=20$; Dmel: $n=28 ;$ Dmoj: $n=23 ;$ Dper: $n=21 ;$ Dpse: $n=16 ;$ Dsec: $n=27 ;$ Dwil: $n=27 ;$ Dere: $n=22 ;$ Dmau: $n=26 ;$ Dana: $n=23 ;$ and Dyak: $n=20$. Sample numbers for data at $24^{\circ} \mathrm{C}$ were the same as Fig. 3C, D. The red lines showed the linear regression functions, and the shaded areas represented the $95 \%$ confidence bands. The point estimates of the Pearson correlation and their $95 \%$ confidence intervals were 0.17 and $[-0.48$, 0.70] in $\mathbf{A}$ and $-0.17,[-0.70,0.48]$ in $\mathbf{B}, 0.17,[-0.48,0.70]$ in $\mathbf{C}$, and $-0.50,[-0.84,0.15]$ in $\mathbf{D}$. E Backward crawling probability at $40^{\circ} \mathrm{C}$ of each species. Probability \pm standard error based on the binomial distribution was shown. Sample numbers in $\mathbf{E}$ were as follows: Dvir: $n=20 ; D m e l: n=$ 28; Dmoj: $n=23$; Dper: $n=22$; Dpse: $n=14 ;$ Dsec: $n=27$; Dwil: $n=27$; Dere: $n=23$; Dmau: $n=26$; Dana: $n=23$; and Dyak: $n=21$

matrix of eight parameters (the bend probability at 24 , 32 , and $40^{\circ} \mathrm{C}$; the crawling speed at 24,32 , and $40^{\circ} \mathrm{C}$; backward crawling probability at $40^{\circ} \mathrm{C}$, and the body length), for each of the 11 Drosophila species. We assumed that these parameters evolved under a multivariate Brownian-motion model [51-53] (see the "Methods" section for details). We estimated the three factors (the evolution rates at phylogenetic trees, the relative evolution rates among the kinematic parameters, and the correlation between the parameter evolutions) by running a Markov chain Monte Carlo simulation.

The phylogenetic analyses indicated that the rates of evolution in the kinematics were highly diverse over branches in the phylogenetic tree (Fig. 11A and Additional file 1: Supplementary Figure 7). In addition, the evolutionary rates were distinct among the eight kinematics parameters (Fig. 11B). The bend probability at $24^{\circ} \mathrm{C}$ and the probability of backward crawling at $40^{\circ} \mathrm{C}$ had a relatively high evolution rate, which might reflect the large diversification of these two parameters among the species (Figs. 9A and 10E).

Some kinematics traits might have evolved cooperatively. To test this possibility, we calculated the correlation of the evolutionary changes between the kinematic parameters. In the correlation analyses of all pairs of the parameters, the crawling speed at $24{ }^{\circ} \mathrm{C}$ and the crawling speed at $32^{\circ} \mathrm{C}$ were the most correlated (Fig. 11C). On the other hand, the evolutions of the bend probability between at 24 and $32{ }^{\circ} \mathrm{C}$ were less correlated. This observation was consistent with the notion that while changes in the bend probability from 24 to $32^{\circ} \mathrm{C}$ among the species were diverse (Fig. 7C), all the species showed an increase in the crawling speed by the temperature shift (Fig. 8C). Consequently, the phylogenetic analyses implied that the kinematics indices of larval locomotion 


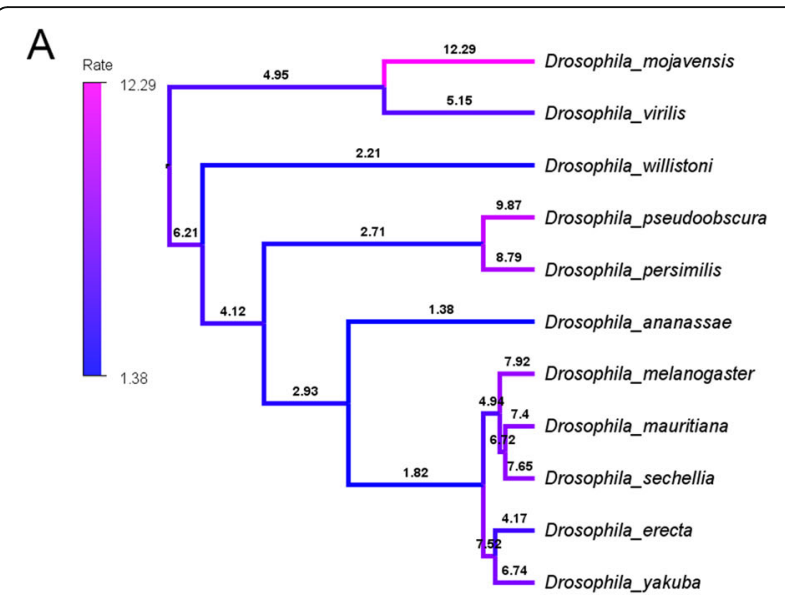

B

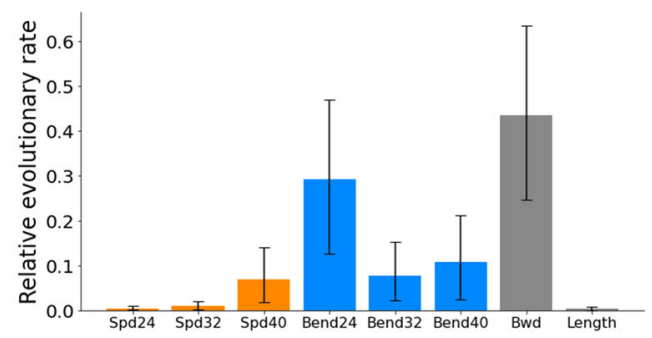

C

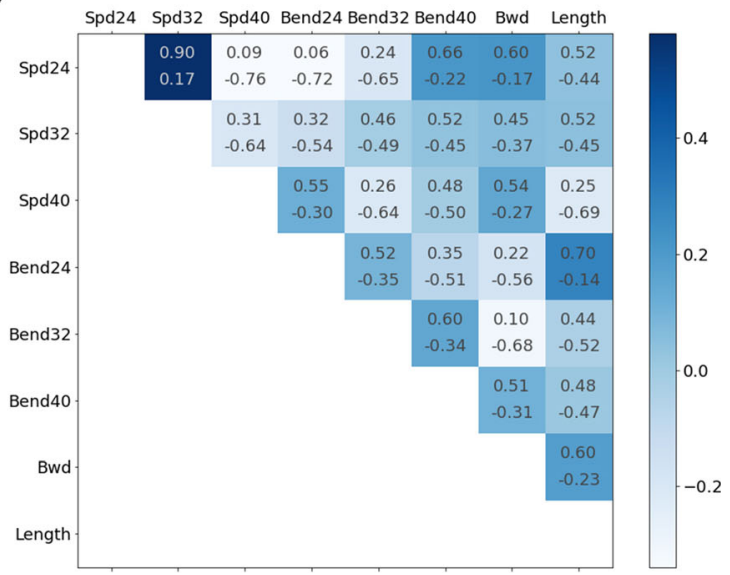

Fig. 11 Phylogenetic analyses of larval locomotion in the genus Drosophila. A Phylogenetic tree estimated from Bayesian inference. The topology was inferred by coding sequences of eight genes of the 11 Drosophila species. As a prior distribution for the Bayesian inference, an uncorrelated exponential (UCED) relaxed-clock model was used. The colour and the value of each branch denoted the relative rate of the evolution of the eight kinematic traits. B Relative evolution rates of the eight kinematics traits estimated by the Bayesian inference. The mean $\pm 95 \%$ highest posterior density was shown. C Correlation between the evolution of eight traits. Numbers represented the range of the $95 \%$ highest posterior density. Abbreviations in $\mathbf{B}$ and $\mathbf{C}$ were as follows: Spd24, crawling speed at $24^{\circ} \mathrm{C}$; Spd 32 , crawling speed at $32^{\circ} \mathrm{C}$; spd40, crawling speed at $40{ }^{\circ} \mathrm{C}$; Bend 24 , bend probability at $24^{\circ} \mathrm{C}$; Bend32, bend probability at $32^{\circ} \mathrm{C}$; Bend 40 , bend probability at $40^{\circ} \mathrm{C}$; Bwd, probability of backward crawling; Length, axial body length of larvae evolved differently in distinct branches of a phylogenetic tree with keeping a correlation between specific locomotion traits such as crawling speed at distinct temperatures.

\section{Discussion}

In this work, we investigated the interspecies differences in larval locomotion in the genus Drosophila. We used bend probability and crawling speed as measures to examine larval locomotion. Despite the similar appearance of larval bodies in different species, the kinematics of larval locomotion is diverged (Fig. 2). The body length is not a leading factor for the diversity of kinematics (Additional file 1: Supplementary Figure 2). The phylogenetic relationship is also not a major determinant for the kinematics (Figs. 3E and 11A). Considering a previous study showing that phylogenetic relationship does not correlate with the divergence in the morphology of larval neuromuscular junctions [44], genetic drift with random accumulation of neutral mutations is unlikely to underlie the divergence in the larval crawling patterns. In contrast, habitat temperature correlates with both the bend probability and crawling speed at both $24^{\circ} \mathrm{C}$ and $32{ }^{\circ} \mathrm{C}$ ambient temperature (Fig. 12), which implies the kinematics indices are adapted to ambient temperature in evolution. Phylogenetic analysis by Bayesian inference suggests that the rates of evolution are divergent among the branches of the phylogenetic tree (Fig. 11A). Among the kinematic parameters, the bend probability at the ambient temperature of $24^{\circ} \mathrm{C}$ and the backward crawling probability at $40^{\circ} \mathrm{C}$ have relatively higher evolution rates than the others, and the evolution of the crawling speed at $24^{\circ} \mathrm{C}$ and $32{ }^{\circ} \mathrm{C}$ is correlated (Fig. $11 \mathrm{~B}, \mathrm{C}$ ). Regarding the questions raised in the "Background" section, our results suggest the following: (1) locomotion kinematics of larvae is divergent among Drosophila species, and (2) the habitat temperature is more related to the kinematics indices (the bend probability and crawling speed) than the body length (Fig. 12).

To conduct the quantitative analyses, we measured the larval crawling behaviour in simple experimental conditions: at the constant temperatures and humidity levels and on a flat surface of agarose gel. However, the environmental conditions in the wild for Drosophila larvae are far more complicated and diverse [8]. For example, Dmoj breeds in cacti in the desert while Dvir inhabits in the slime fluxes in temperate and subarctic climates (Additional file 1: Supplementary Figure 3 [8];). Our data in this paper imply that the variation of the kinematics should be related to the diversity of environmental conditions including habitat temperature. Comprehensive and quantitative analyses of larval locomotion in nature would shed light on the causes and meanings of the 


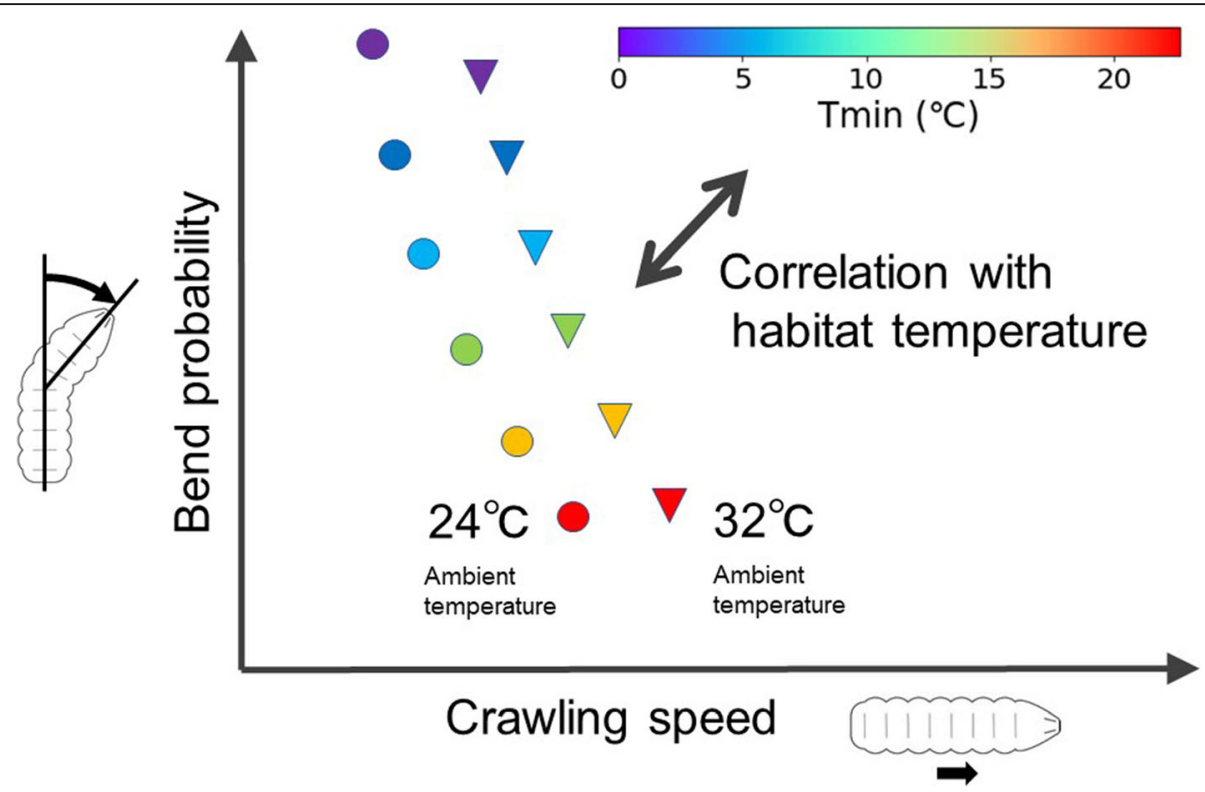

Fig. 12 Summary of this study. Disks and triangles denoted the kinematics of each species at $24^{\circ} \mathrm{C}$ and $32{ }^{\circ} \mathrm{C}$, respectively

changes in larval kinematics that appeared in the evolution.

The correlation between habitat temperature and the kinematics raises a question about the underlying mechanisms of adaptive change in kinematics to the habitat temperature. We presume that the adaptive change was likely to be driven by two factors: the pupation positioning and competition over food. Regarding the pupation behaviour, a previous study showed a correlation between path lengths of crawling and heights of pupation location in D. melanogaster [16]. Since pupae are immobile, adequate selection of pupation position is vital for their survival. Moisture is one of the key factors that affects the selection of pupation site, since dry environments can cause pupal desiccation, while soaked media might lead to the drowning of pupae [16, 54]. It is reasonable to assume that habitat temperature is a leading factor in determining the moisture levels of microenvironments of Drosophila larvae. Accordingly, divergent temperatures in the habitats of flies lead to variability in moisture levels, the ambient moisture affects the pupation position [54], and the pupation position is related to the path length of crawling [16]. These three links might underlie the relationship between the larval locomotion kinematics and habitat temperature of each species.

The second possible mechanism underlying the relation between habitat temperature and locomotion kinematics is related to competition over food. It is suggested that larval feeding is competitive between individuals [55]. There is a positive relation between fitness and density (called Allee effects) in D. melanogaster larvae [56], and so female flies aggregate eggs when laying them [55]. Consequently, the larval population tends to be overcrowded [57], and larvae compete over the limited resources to meet their need to consume sufficient amounts of nutrients within limited larval periods [58]. If the available food is limited and larval density is high, larvae need to crawl further [17], which can be an evolutionary driving force to increase the crawling distance. In contrast, if there is plenty of food in the habitat, the crawling distance remains unchanged or even decreases during evolution, because crawling behaviour is energetically costly [14]. Consistent with this implication, the diversification in the larval kinematics in the 11 Drosophila species gives rise to the variation in the crawling distance (Fig. 5). Accordingly, feeding conditions, especially the choice of which foods to eat, can affect the kinematics of larval locomotion in evolutionary processes so that the divergence of food to eat can lead to the divergence in locomotion kinematics. In regard to this point, food for larvae of the genus Drosophila is divergent and related to their living environments [8-10]. So, the nutritional conditions (nutrient balances, fermentation, etc.) and physical properties (hardness, wetness, etc.) of food to eat vary among the Drosophila species, which can drive evolutionary divergence in the kinematics of larval locomotion. Then, the growth of plants, including fruits and vegetables, for larvae is strongly affected by habitat temperature. Therefore, divergence in habitat temperature may affect the locomotion kinematics during evolution through divergence in the foods that larvae feed on, and divergence in requisite feeding behaviour for larval growth. Comprehensive kinematics studies of other Drosophila species and 
quantitative analyses of microenvironments of wild larvae in nature will give us insights into the relationships between ambient temperature and diverse larval locomotion kinematics.

How can we approach the neural circuit mechanisms underlying the interspecies divergence in larval locomotion? Circuit mechanisms in larval locomotion have been examined intensively in Drosophila melanogaster. Recent connectomics studies have identified several key neurons for larval locomotion in Drosophila melanogaster [20, 30, 59-67]. Regarding bending, the thoracic neuromere was shown to be important in bending in chemotaxis [30]. Especially, the commissural connection is crucial for bending [68]. In addition, a signal from the chordotonal sensory organ is also required to generate bending [69]. Regarding the crawling speed, a group of inhibitory premotor neurons was identified to be critical [64]. Proprioceptive feedback and neuromodulation are both important for the normal crawling speed [12, 63]. Furthermore, the kinematics of the larval locomotion have been measured and investigated quantitatively in detail $[26,31,33,34,70-73]$ and analysed by mathematical modelling [74-78]. Regarding temperature sensing, neuronal and molecular mechanisms on temperature-guided behaviour have been clarified [22-24, 27, 79-83]. These extensive findings on the cellular and molecular mechanisms on larval crawling in Drosophila melanogaster will be an ideal starting point to investigate the evolution of larval behaviour in the genus Drosophila. For example, differences in commissure fibre tracts in the central nervous system [68] among the species might underlie the divergence in the bend probability. Interspecies comparison of a group of interneurons PMSIs (period-positive median segmental interneurons), which are involved in the crawling speed [64], would reveal the neural mechanisms on the evolutionary diversification in the crawling speed. Comparative analyses of neural network architectures and gene expression among the Drosophila species will relate the evolution of the nervous system to the adaptive diversification in larval behaviour.

\section{Conclusions}

Here, our work suggests interspecific diversity of the larval locomotion kinematics among species in the genus Drosophila. The variation is not correlated to the body length but rather to the habitat temperature of the species: larvae of species inhabiting moderate-temperature environments exhibited low bend probability and fast crawling, or long crawling distance, whereas those inhabiting low-temperature environments showed frequent bending and slow crawling, or short crawling distance.
Phylogenetic analyses based on Bayesian inference indicate that the evolutionary rate of the kinematic properties is diverse among phylogenetic tree branches. These results suggest that the kinematics of larval locomotion in the genus Drosophila diverged under the effects of the ambient temperature of their habitats.

\section{Methods \\ Drosophila strains}

We used the following fly stocks (17 strains from 11 species): Drosophila ananassae (k-s01), Drosophila erecta (k-s02), Drosophila yakuba (k-s03), Drosophila melanogaster (k-s04), Drosophila sechellia (k-s10), Drosophila persimilis (k-s11), Drosophila pseudoobscura (k-s12), Drosophila willistoni (k-s13), Drosophila virilis (k-s14), and Drosophila mojavensis (k-s15), Drosophila melanogaster collected at Kyoto (k-aba029) and Iriomote (kaba032), Drosophila ananassae collected at Ogasawara (k-aaa027) and Iriomote (k-aaa309), and Drosophila virilis collected at Hokkaido (E-15601) and Toyama (E15605) from KYORIN-Fly, Fly Stocks of Kyorin University and Drosophila mauritiana (\#900020) from KYOTO Stock Center (DGRC) at the Kyoto Institute of Technology. These strains have been maintained at $23^{\circ} \mathrm{C}$ (except for Drosophila persimilis at $20^{\circ} \mathrm{C}$ ) in the stock centres for more than 10 years. All animals were raised on standard cornmeal-based food at $25^{\circ} \mathrm{C}$ in the authors' laboratory before the experiments for less than 3 months after the acquisition from the stock centres.

\section{Recording larval locomotion}

Third-instar wandering larvae of each strain were picked up and gently washed in deionised water. Residual food on the larvae was brushed off with a paintbrush. An agarose stage $($ size $9 \mathrm{~cm} \times 9 \mathrm{~cm} \times 5$ $\mathrm{mm} ; 1.5 \%$ agarose; RIKAKEN STAR agarose powder \#RSV-AGRP-100G) was placed on a temperaturecontrolled plate (Cool Plate, AS ONE Corporation, Japan), and the temperature of the surface was kept at $24 \pm 1{ }^{\circ} \mathrm{C}, 32 \pm 1{ }^{\circ} \mathrm{C}$, or $40 \pm 1{ }^{\circ} \mathrm{C}$. Eight to ten larvae were placed on the agarose arena with a paintbrush. The larvae were illuminated with infrared light, which larvae cannot see (LDQ-150IR2-850, wavelength $=850 \mathrm{~nm}$, CCS Inc., Japan) and recorded at five frames/s with a CCD camera (CGE-B013-U, MIGHTEX, Canada; resolution 1280 pixels $\times 960$ pixels) and an infrared filter (775LP filter, Omega Optical, USA), and saved as a series of bitmap files. In our setting, the scale of the image is $0.13 \mathrm{~mm} /$ pixel. For each of the 17 strains (from 11 species) and at each of the three ambient temperatures, we repeated the measurement three times. 


\section{Larva tracking}

We obtained a time series of bitmap image files by the procedure described above. The bitmap files were converted to tiff format by Fiji (https://imagej.net/fiji). In cases where larvae accumulated at the first frame, we removed the first several images until the larvae dispersed enough to be identified individually. The $x$ and $y$ coordinates of the centroid and the bend angle (Fig. 1E) of individual larvae were obtained by using the FIMTrack software [28]. We used tracking data of single larvae that were continuously tracked for more than 300 frames (which corresponds to $1 \mathrm{~min}$ ). For the intraspecific analysis in Fig. 4, single larvae with more than 150 continuous frames were tracked. In case a larva collided with another larva in the middle of the recording and the larva was traced differently before and after the collision with distinct labels, we treated the two traces as two larvae. The crawling speed of each larva was obtained as the median of crawling speed in the whole trace of the larva. Bend probability of each larva was calculated as the ratio of the number of frames showing bending (Fig. 3 for the definition of bending) to the total number of the frames of its trace. The data of the three movies for each species/strain were merged. The coordinates and bend angles were smoothed and plotted by Python 3.7. For the visualisation of the traces in Fig. 1E, F, we smoothed the data by a uniform window function with five frames. The number of pixels in a single larva ranges from about 150 (Dyak) to 500 (Dpse) that is sufficient to capture the body bend angles.

\section{Clustering analysis of kinematics}

For clustering analysis of the plots in Fig. 2A, we made a probability distribution. We discretised the centroid speed axis by an interval of $0.1 \mathrm{~mm} / \mathrm{s}$ between 0 and 4 $\mathrm{mm} / \mathrm{s}$ and the bend angle axis by an interval of two degrees between 0 and $140^{\circ}$, then created 2800 bins in total (40 bins in the speed axis and 70 bins in the bend angle axis). For each scatter plot, we counted the number of points in each bin and obtained probability density by normalisation with the total number of points. We calculated the Kullback-Leibler divergence (KL) of probability distributions of two species $p$ (bin) and $q$ (bin) as follows:

$$
\mathrm{KL}(p, q)=\sum_{\mathrm{bin}=1}^{2800} p(\mathrm{bin}) * \log \frac{p(\mathrm{bin})}{q(\mathrm{bin})} .
$$

Since $\mathrm{KL}(p, q)$ is an asymmetric measure $(\mathrm{KL}(p, q) \neq$ $\mathrm{KL}(q, p))$, we calculated the Jensen-Shannon divergence (JS), which is a symmetric alternative to the KullbackLeibler divergence:

$$
\mathrm{JS}(p, q)=\frac{1}{2}\{\mathrm{KL}(p, q)+\mathrm{KL}(q, p)\}=\mathrm{JS}(q, p) .
$$

Based on the Jensen-Shannon divergence, we conducted the hierarchical clustering using Python 3.7 and the SciPy library.

\section{Habitat temperature of the Drosophila species}

Global climate data were obtained from the WorldClim data website (https://www.worldclim.org/data/ worldclim21.html). We used minimum, mean, and maximum temperature in the world at a spatial resolution of $5 \min (9.25 \mathrm{~km} \times 9.25 \mathrm{~km})$. Habitat regions of each Drosophila species were obtained from the literature [7, 41, 42] and the DrosWLD-Species database (https://bioinfo. museum.hokudai.ac.jp/db/modules/stdb/index. php?action=tbl\&tbl_id=21) and traced them on the world map (2160 pixels $\times 4320$ pixels) obtained from the WorldClim by using Adobe Illustrator software (Adobe Inc., USA). The maps in Additional file 1: Supplementary Figure 2 were generated by Adobe Illustrator, and the statistics of the temperature within each species' habitat were calculated by Python 3.7.

\section{Statistical analysis}

Statistical calculation was conducted by Python 3.7 in the following analyses: Kruskal-Wallis test in Fig. 3C, D, Pearson correlation in Figs. 6B, C; 7B, C; 8B, C; and Additional file 1: Figure $2 \mathrm{~B}, 2 \mathrm{C}, 4 \mathrm{~B}$ and $5 \mathrm{~B}$, and MannWhitney $U$ test in Fig. 7A and 8A and Additional file 1: Supplementary Figure 5A and 6A.

\section{Phylogenetic analyses}

We adopted Bayesian statistics for phylogenetic analyses. In all the phylogenetic analyses, we used the software package RevBayes v.1.0.7 [29].

We estimated the Drosophila phylogenetic trees (Fig. $8 \mathrm{~A}$ ) by using eight nuclear loci from Turelli et al. [50]: aldolase, bicoid, enolase, esc, transaldolase, white, wingless, and yellow. We used Scaptodrosophila lebanonensis (S. lebanonensis) as an outgroup. Coding sequences of these genes for all the species except for D. mauritiana and S. lebanonensis were obtained from Kalay et al. [51]. Coding sequences of the genes of $D$. mauritiana and $S$. lebanonensis were obtained from the NCBI website (https://www.ncbi.nlm.nih.gov/). The genes were aligned with MAFFT version 7 [84]. We selected the eight genes out of twenty genes described in Turelli et al. [50] because homologous genes of the other twelve genes were not identified in the genome assembly of $D$. mauritiana. Based on the eight coding sequences of the twelve species, we performed chronogram analyses described in Turelli et al. [50] and obtained the phylogenetic tree in Fig. 8A, where the root is the branch of S. lebanonensis 
and the length of each branch indicates the relative time in the evolution. We repeated the phylogeny inference four times to obtain four trees to check the robustness of this inference in the following analysis.

Based on the phylogenetic tree obtained above, we analysed the evolution of kinematics parameters. We used eight parameters (bend probability at 24,32 , and $40^{\circ} \mathrm{C}$; crawling speed at 24,32 , and $40^{\circ} \mathrm{C}$; backward crawling probability at $40^{\circ} \mathrm{C}$; and body length), for each of the 11 Drosophila species. In this analysis, while the topology of the phylogenetic tree is unchanged, the rates of evolution of the kinematic parameters and the rates of evolution of the branches of the phylogenetic tree were estimated by Bayesian inference. We assumed the kinematics parameters evolve under a multivariate Brownian-motion model $[52,53]$. This model consists of two components: the relative rates of evolution among the kinematics parameters and the correlation between each pair of the kinematics parameters [51]. The Brownian model assumes that the changes in the kinematics parameters during evolution are additive, which has two consequences: (1) parameters can become negative, and (2) evolution rates can depend on the scale of the kinematics parameters. To cope with these properties, we adopted log-transformation to the kinematics parameters as described previously [51]. This transformation guarantees that the original kinematics parameters (which are exponentials of the log-transformation) remain positive and controls the size issue by transforming additive changes to multiplicative ones [51]. For prior models for the evolution rates of the branches, we specified an uncorrelated exponential (UCED) relaxed-clock model, where the rate of each branch is drawn independently from an exponential distribution. We ran the Markov chain Monte Carlo (MCMC) simulation of UCED with each of the four phylogenetic trees described in the previous paragraph (Fig. 11). Consistent results were obtained by another prior model, an uncorrelated gamma (UCG) relaxed-clock model (Supplementary Figure 7), which indicates the predictions are robust to the choice of priors.

\section{Supplementary Information}

The online version contains supplementary material available at https://doi. org/10.1186/s12915-021-01110-4.

Additional file 1: Figure S1. The negative correlation between the crawling speed and bend probability is robust to the angle threshold for the definition of bend. (A-C) Scatter plots of the bend probability at $24^{\circ} \mathrm{C}$ against the crawling speed at $24^{\circ} \mathrm{C}$. (A) The angle threshold for bend was 10 degrees. (B) The threshold was 20 degrees. (C) The threshold was 30 degrees. Median \pm sem was shown. B was the same panel as Figure $3 C$ represented here for comparison. (D) Scatter plot of the median of absolute bend angle at $24^{\circ} \mathrm{C}$ against the crawling speed at $24^{\circ} \mathrm{C}$. The red lines showed the linear regression functions and the shaded areas represented the $95 \%$ confidence band. The point estimates of the
Pearson correlation and its 95\% confidence intervals were -0.80 and $[-0.95,-0.39]$ in $A,-0.76$ and $[-0.94,-0.30]$ in $B,-0.71$ and $[-0.92,-0.19]$ in $C$, and -0.66 and $[-0.90,-0.11]$ in D. Figure S2. Interspecies comparison between larva length and kinematics parameters. (A) Axial body length of larvae of the 11 species. Sample numbers were the following: Dvir: $n=69$; Dmel: $n=72$; Dmoj: $n=82$; Dper: $n=76$; Dpse: $n=60$; Dsec: $n=68$; Dwil: $n=$ 66; Dere: $n=69$; Dmau: $n=69$; Dana: $n=68$; Dyak: $n=56$. (B) Scatter plot of the bend probability at $24^{\circ} \mathrm{C}$ against the larva length of the 11 species. (C) Scatter plot of the crawling speed at $24^{\circ} \mathrm{C}$ against the larva length of the 11 species. In B and C, median \pm sem was shown. The red lines showed the linear regression functions and the shaded areas represented the $95 \%$ confidence band. The point estimates of the Pearson correlation and its $95 \%$ confidence intervals were 0.55 and $[-0.07,0.87]$ in $B$ and 0.04 and $[-0.57,0.63]$ in C. Figure S3. World maps of histograms of habitat temperature of the Drosophila species. The top of each panel showed a map of the habitat of the labelled species. The bottom on each panel showed a histogram of minimum (green) and maximum (magenta) temperatures within the habitat. Asterisks denoted the warmest peak in the minimum habitat temperature. The temperature at the peak was called Tmin in this study. Figure S4. Relationship between the kinematics of larval locomotion and the range of habitat temperature of the Drosophila species (A) Scatter plot of the bend probability speed at $24^{\circ} \mathrm{C}$ against the range of habitat temperature of the 11 species. (B) Scatter plot of the crawling speed probability at $24^{\circ} \mathrm{C}$ against the larva length of the 11 species. Median \pm sem was shown. The red lines showed the linear regression functions and the shaded areas represented the $95 \%$ confidence band. The point estimates of the Pearson correlation and its $95 \%$ confidence intervals were 0.65 and $[0.08,0.90]$ in A and -0.69 and $[-0.91,-0.15]$ in B. Figure S5. Bend probability in the 11 Drosophila species at $40^{\circ} \mathrm{C}$. (A) Bend probability of the 11 species at $32^{\circ} \mathrm{C}$ and $40^{\circ} \mathrm{C}$. The data at $32^{\circ} \mathrm{C}$ were the same as in Figure $4 \mathrm{~A}$. (B) Scatter plot of the bend probability at $40^{\circ} \mathrm{C}$ against at $32^{\circ} \mathrm{C}$. Sample numbers at $40^{\circ} \mathrm{C}$ were the following: Dvir: $n=20$; Dmel: $n=28$; Dmoj: $n=23$; Dper: $n=21$; Dpse: $n=$ 16; Dsec: $n=27$; Dwil: $n=27$; Dere: $n=22 ;$ Dmau: $n=26$; Dana: $n=23$; Dyak: $\mathrm{n}=20$. Sample numbers at $32^{\circ} \mathrm{C}$ were described in the legend of Figure 4. Median \pm sem was shown in B. Figure S6. Crawling speed in the 11 Drosophila species at $40^{\circ} \mathrm{C}$. (A) Crawling speed of the 11 species at $32^{\circ} \mathrm{C}$ and $40^{\circ} \mathrm{C}$. The data at $32^{\circ} \mathrm{C}$ were the same as in Figure $5 \mathrm{~A}$. (B) Scatter plot of the crawling speed at $40^{\circ} \mathrm{C}$ against at $32^{\circ} \mathrm{C}$. Sample numbers at $40^{\circ} \mathrm{C}$ were the following: Dvir: $n=20$; Dmel: $n=28$; Dmoj: $n=23$; Dper: $n=$ 21; Dpse: $n=16$; Dsec: $n=27$; Dwil: $n=27$; Dere: $n=22 ;$ Dmau: $n=26$; Dana: $n=23$; Dyak: $n=20$. Sample numbers at $32^{\circ} \mathrm{C}$ were described in the legend of Figure 5. Median \pm sem was shown in B. Figure S7. Phylogenetic analyses of larval locomotion based on distinct prior models. (A) Phylogenetic tree estimated from Bayesian inference with an uncorrelated exponential (UCED) relaxed-clock model as the prior. Values of each branch denoted the range of $95 \%$ highest posterior density of the inference of the evolution rates shown in Figure 11A. ( $B$ and $C$ ) Phylogenetic tree estimated from Bayesian inference with an uncorrelated gamma (UCG) relaxed clock model. Values of each branch showed the evolution rate (B) and its range of $95 \%$ highest posterior density (C).

\section{Acknowledgements}

We thank KYORIN-Fly, Fly Stocks of Kyorin University and KYOTO Stock Center (DGRC) at the Kyoto Institute of Technology for providing fly lines, and Dr. Takashi Makino for the advice about climate data in the world. We thank Ms. Shibahara for tracing the habitats of the 11 species. We thank Dr. Masayoshi Watada for the critical comments on the paper.

\section{Authors' contributions}

YM recorded the larval locomotion and analysed the data by the FIMTrack software. AN edited the manuscript. HK conceived this project, supervised the work, recorded the larval locomotion, analysed the data by FIMTrack, performed the statistical analyses, and wrote the manuscript. All authors read and approved the final manuscript.

Funding

This work was supported by MEXT/JSPS KAKENHI grants (17K19439, $19 \mathrm{H} 04742$, and $20 \mathrm{H} 05048$ to A.N. and $17 \mathrm{K07042}$ and $20 \mathrm{~K} 06908$ to H.K.). 


\section{Availability of data and materials}

All data generated or analysed during this study are included in this article, its supplementary information file, or in the figshare repository: https://doi. org/10.6084/m9.figshare.15041829 [85]

\section{Declarations}

Ethics approval and consent to participate

Not applicable.

\section{Consent for publication}

Not applicable.

\section{Competing interests}

The authors declare that they have no competing interests.

\section{Author details}

'Department of Complexity Science and Engineering, Graduate School of Frontier Science, The University of Tokyo, 5-1-5 Kashiwanoha, Kashiwa, Chiba 277-8561, Japan. ${ }^{2}$ Department of Physics, Graduate School of Science, The University of Tokyo, 7-3-1 Hongo, Bunkyo-ku, Tokyo 133-0033, Japan. ${ }^{3}$ School of Informatics and Engineering, The University of Electro-Communications, 1-5-1, Chofugaoka, Chofu-shi, Tokyo 182-8585, Japan.

Received: 25 June 2021 Accepted: 29 July 2021

Published online: 02 September 2021

\section{References}

1. Alexander RM. Principles of animal locomotion. Princeton: Princeton University Press; 2006.

2. Kappeler P. Animal behaviour: evolution and mechanisms. Berlin: Springer; 2010. https://doi.org/10.1007/978-3-642-02624-9.

3. Theunissen LM, Bekemeier HH, Dürr V. Comparative whole-body kinematics of closely related insect species with different body morphology. J Exp Biol. 2015;218(Pt 3):340-52. https://doi.org/10.1242/jeb.114173.

4. Fuller PO, Higham TE, Clark AJ. Posture, speed, and habitat structure: threedimensional hindlimb kinematics of two species of padless geckos. Zoology. 2011;114(2):104-12. https://doi.org/10.1016/j.zool.2010.11.003.

5. Stoessel A, Fischer MS. Comparative intralimb coordination in avian bipedal locomotion. J Exp Biol. 2012;215(Pt 23):4055-69. https://doi.org/10.1242/jeb. 070458.

6. Thompson NE, O'Neill MC, Holowka NB, Demes B. Step width and frontal plane trunk motion in bipedal chimpanzee and human walking. J Hum Evol. 2018;125:27-37. https://doi.org/10.1016/j.jhevol.2018.09.006.

7. Markow TA, O'Grady PM. Drosophila: a guide to species identification and use. Cambridge: Academic Press; 2005.

8. Markow. The secret lives of Drosophila flies. Elife. 2015:4:1-9.

9. Watada M, Hayashi Y, Watanabe K, Mizutani S, Mure A, Hattori Y, et al. Divergence of Drosophila species: longevity and reproduction under different nutrient balances. Genes to Cells. 2020;1-7.

10. Watanabe K, Kanaoka Y, Mizutani S, Uchiyama H, Yajima S, Watada M, et al Interspecies comparative analyses reveal distinct carbohydrate-responsive systems among Drosophila species. Cell Rep. 2019;28:2594-2607.e7. https:// doi.org/10.1016/j.celrep.2019.08.030.

11. Green $\mathrm{CH}$, Burnet B, Connolly KJ. Organization and patterns of inter- and intraspecific variation in the behaviour of Drosophila larvae. Anim Behav. 1983;31(1):282-91. https://doi.org/10.1016/S0003-3472(83)80198-5.

12. Fox LE, Soll DR, Wu CF. Coordination and modulation of locomotion pattern generators in Drosophila larvae: effects of altered biogenic amine levels by the tyramine $\beta$ hydroxlyase mutation. J Neurosci. 2006;26(5):1486-98. https://doi.org/10.1523/JNEUROSCI.4749-05.2006.

13. Berrigan D, Pepin DJ. How maggots move: allometry and kinematics of crawling in larval Diptera. J Insect Physiol. 1995;41(4):329-37. https://doi. org/10.1016/0022-1910(94)00113-U.

14. Berrigan D, Lighton JR. Bioenergetic and kinematic consequences of limblessness in larval Diptera. J Exp Biol. 1993;179(1):245-59. https://doi. org/10.1242/jeb.179.1.245.

15. Ruiz-Dubreuil G, Burnet B, Connolly K, Furness P. Larval foraging behaviour and competition in Drosophila melanogaster. Heredity (Edinb). 1996;76(1): 55-64. https://doi.org/10.1038/hdy.1996.7.
16. Sokolowski MB. Genetics and ecology of Drosophila melanogaster larval foraging and pupation behaviour. J Insect Physiol. 1985;31(11):857-64. https://doi.org/10.1016/0022-1910(85)90103-9.

17. Sokolowski MB, Pereira HS, Hughes K. Evolution of foraging behavior in drosophila by density-dependent selection. Proc Natl Acad Sci U S A. 1997; 94(14):7373-7. https://doi.org/10.1073/pnas.94.14.7373.

18. Venken KJT, Simpson JH, Bellen HJ. Genetic manipulation of genes and cells in the nervous system of the fruit fly. Neuron. 2011;72(2):202-30. https://doi. org/10.1016/j.neuron.2011.09.021.

19. Simpson $\mathrm{JH}$, Looger LL. Functional imaging and optogenetics in Drosophila. Genetics. 2018;208(4):1291-309. https://doi.org/10.1534/genetics.117.300228.

20. Schneider-Mizell CM, Gerhard S, Longair M, Kazimiers T, Li F, Zwart MF, et al. Quantitative neuroanatomy for connectomics in Drosophila. Elife. 2016;5:136.

21. Ohyama T, Schneider-Mizell CM, Fetter RD, Aleman JV, Franconville R, Rivera-Alba M, et al. A multilevel multimodal circuit enhances action selection in Drosophila. Nature. 2015;520(7549):633-9. https://doi.org/10.103 8/nature14297.

22. Liu L, Yermolaieva O, Johnson WA, Abboud FM, Welsh MJ. Identification and function of thermosensory neurons in Drosophila larvae. Nat Neurosci. 2003;6(3):267-73. https://doi.org/10.1038/nn1009.

23. Rosenzweig M, Brennan KM, Tayler TD, Phelps PO, Patapoutian A, Garrity PA The Drosophila ortholog of vertebrate TRPA1 regulates thermotaxis. Genes Dev. 2005;19(4):419-24. https://doi.org/10.1101/gad.1278205.

24. Kwon Y, Shen WL, Shim HS, Montell C. Fine thermotactic discrimination between the optimal and slightly cooler temperatures via a TRPV channel in chordotonal neurons. J Neurosci. 2010;30(31):10465-71. https://doi.org/10.1 523/JNEUROSCI.1631-10.2010.

25. Ohyama T, Jovanic T, Denisov G, Dang TC, Hoffmann D, Kerr RA, et al. Highthroughput analysis of stimulus-evoked behaviors in Drosophila larva reveals multiple modality-specific escape strategies. PLoS One. 2013;8(8): e71706. https://doi.org/10.1371/journal.pone.0071706.

26. Luo L, Gershow M, Rosenzweig M, Kang K, Fang-Yen C, Garrity PA, et al. Navigational decision making in Drosophila thermotaxis. J Neurosci. 2010; 30(12):4261-72. https://doi.org/10.1523/JNEUROSCI.4090-09.2010.

27. Klein M, Afonso B, Vonner AJ, Hernandez-Nunez L, Berck M, Tabone CJ, et al Sensory determinants of behavioral dynamics in Drosophila thermotaxis. Proc Natl Acad Sci U S A. 2015;112(2):E220-9. https://doi.org/10.1073/pna S. 1416212112.

28. Risse B, Berh D, Otto N, Klämbt C, Jiang X. FIMTrack: an open source tracking and locomotion analysis software for small animals. PLoS Comput Biol. 2017;13:1-15.

29. Hohna S, Landis MJ, Heath TA, Boussau B, Lartillot N, Moore BR, et al. RevBayes: Bayesian phylogenetic inference using graphical models and an interactive model-specification language. Syst Biol. 2016;65(4):726-36. https://doi.org/10.1093/sysbio/syw021.

30. Tastekin I, Riedl J, Schilling-Kurz V, Gomez-Marin A, Truman JW, Louis M. Role of the subesophageal zone in sensorimotor control of orientation in drosophila larva. Curr Biol. 2015;25(11):1448-60. https://doi.org/10.1016/j.cub.2015.04.016.

31. Gomez-Marin A, Stephens GJ, Louis M. Active sampling and decision making in Drosophila chemotaxis. Nat Commun. 2011;2(1):441. https:/doi. org/10.1038/ncomms1455.

32. Berni J, Pulver SR, Griffith LC, Bate M. Autonomous circuitry for substrate exploration in freely moving Drosophila larvae. Curr Biol. 2012;22(20):186170. https://doi.org/10.1016/j.cub.2012.07.048.

33. Gershow M, Berck M, Mathew D, Luo L, Kane EA, Carlson JR, et al. Controlling airborne cues to study small animal navigation. Nat Methods. 2012;9(3):290-6. https://doi.org/10.1038/nmeth.1853.

34. Gomez-Marin A, Louis M. Multilevel control of run orientation in Drosophila larval chemotaxis. Front Behav Neurosci. 2014:8:1-14.

35. Sims DW, Humphries NE, Hu N, Medan V, Berni J. Optimal searching behaviour generated intrinsically by the central pattern generator for locomotion. Elife. 2019;8:1-31. https://doi.org/10.7554/eLife.50316.

36. Clark AG, Eisen MB, Smith DR, Bergman CM, Oliver B, Markow TA, et al. Evolution of genes and genomes on the Drosophila phylogeny. Nature. 2007:450(7167):203-18. https://doi.org/10.1038/nature06341.

37. Miller DE, Staber C, Zeitlinger J, Hawley RS. Highly contiguous genome assemblies of 15 drosophila species generated using nanopore sequencing. G3 Genes, Genomes. Genet. 2018;8:3131-41.

38. Lin J. Divergence measures based on the Shannon entropy. IEEE Trans Inf Theory. 1991;37(1):145-51. https://doi.org/10.1109/18.61115. 
39. Risse B, Thomas S, Otto N, Löpmeier T, Valkov D, Jiang X, et al. FIM, a novel FTRR-based imaging method for high throughput locomotion analysis. PLoS One. 2013;8:1-11.

40. Schmidt-Nielsen K. Animal physiology: adaptation and environment. Cambridge: Cambridge University Press; 1997. https://doi.org/10.1017/9780511 801822

41. Makino T, Kawata M. Habitat variability correlates with duplicate content of drosophila genomes. Mol Biol Evol. 2012;29(10):3169-79. https://doi.org/10.1 093/molbev/mss133.

42. Brake I, Baechli G. Drosophilidae (Diptera). Apollo Books; 2008: https://doi. org/10.1163/9789004261037.

43. Hijmans RJ, Cameron SE, Parra JL, Jones PG, Jarvis A. Very high resolution interpolated climate surfaces for global land areas. Int J Climatol. 2005; 25(15):1965-78. https://doi.org/10.1002/joc.1276

44. Campbell M, Ganetzky B. Extensive morphological divergence and rapid evolution of the larval neuromuscular junction in Drosophila. Proc Natl Acad Sci U S A. 2012;109.

45. Russo CAM, Takezaki N, Nei M. Molecular phylogeny and divergence times of drosophilid species. Mol Biol Evol. 1995;12(3):391-404. https://doi.org/10.1 093/oxfordjournals.molbev.a040214

46. Tracey WD, Tracey WD, Wilson RI, Wilson Rl, Laurent G, Laurent G, et al. Gene essential for nociception. Cell. 2003;113(2):261-73. https://doi.org/10.1 016/S0092-8674(03)00272-1.

47. Petersen M, Tenedini F, Hoyer N, Kutschera F, Soba P. Assaying thermonociceptive behavior in Drosophila IOarvae. Bio-Protocol. 2018;8:1-12.

48. Dason JS, Cheung A, Anreiter I, Montemurri VA, Allen AM, Sokolowski MB. Drosophila melanogaster foraging regulates a nociceptive-like escape behavior through a developmentally plastic sensory circuit. Proc Natl Acad Sci. 2019;201820840.

49. Höhna S, Landis MJ, Heath TA. Phylogenetic inference using RevBayes. Curr Protoc Bioinforma. 2017:6:1-34.

50. Turelli M, Cooper BS, Richardson KM, Ginsberg PS, Peckenpaugh B, Antelope CX, et al. Rapid global spread of wRi-like Wolbachia across multiple Drosophila. Curr Biol. 2018;28:963-971.e8.

51. Kalay G, Atallah J, Sierra NC, Tang AM, Crofton AE, Murugesan MK, et al. Evolution of larval segment position across 12 Drosophila species*. Evolution (N Y). 2020;74:1409-22.

52. Huelsenbeck J, Bruce R. Detecting correlation between characters in a comparative analysis with uncertain phylogeny. Evolution (N Y). 2003:57: 1237-47.

53. Lartillot N, Poujol R. A phylogenetic model for investigating correlated evolution of substitution rates and continuous phenotypic characters. Mol Biol Evol. 2011;28(1):729-44. https://doi.org/10.1093/molbev/msq244.

54. Sokolowski MB, Kent C, Wong J. Drosophila larval foraging behaviour: developmental stages. Anim Behav. 1984;32(3):645-51. https://doi.org/10.1 016/50003-3472(84)80139-6.

55. Rohlfs $M$, Hoffmeister TS. An evolutionary explanation of the aggregation model of species coexistence. Proc R Soc B Biol Sci. 2003;270:33-5.

56. Wertheim B, Marchais J, Vet LEM, Dicke M. Allee effect in larval resource exploitation in Drosophila: an interaction among density of adults, larvae, and micro-organisms. Ecol Entomol. 2002;27(5):608-17. https://doi.org/10.1 046/j.1365-2311.2002.00449.x

57. Burnet B, Sewell D, Bos M. Genetic analysis of larval feeding behaviour in Drosophila melanogaster: II. Growth relations and competition between selected lines. Genet Res. 1977;30(2):149-61. https://doi.org/10.1017/5001 6672300017559 .

58. Nicholson AJ. An outline of the dynamics of animal. Aust J Zool. 1954;2(1): 9-65. https://doi.org/10.1071/ZO9540009.

59. Kohsaka H, Zwart MF, Fushiki A, Fetter RD, Truman JW, Cardona A, et al. Regulation of forward and backward locomotion through intersegmental feedback circuits in Drosophila larvae. Nat Commun. 2019;10(1):2654. https://doi.org/10.1038/s41467-019-10695-y.

60. Carreira-Rosario A, Zarin AA, Clark MQ, Manning L, Fetter RD, Cardona A, et al. MDN brain descending neurons coordinately activate backward and inhibit forward locomotion. Elife. 2018;7:1-28.

61. Fushiki A, Zwart MF, Kohsaka H, Fetter RD, Cardona A, Nose A. A circuit mechanism for the propagation of waves of muscle contraction in Drosophila. Elife. 2016;5:1-23.

62. Heckscher ES, Zarin AA, Faumont S, Clark MQ, Manning L, Fushiki A, et al. Even-skipped+ interneurons are core components of a sensorimotor circuit that maintains left-right symmetric muscle contraction amplitude. Neuron 2015;88(2):314-29. https://doi.org/10.1016/j.neuron.2015.09.009.
63. Hughes $\mathrm{CL}$, Thomas JB. A sensory feedback circuit coordinates muscle activity in Drosophila. Mol Cell Neurosci. 2007;35(2):383-96. https://doi.org/1 0.1016/j.mcn.2007.04.001.

64. Kohsaka H, Takasu E, Morimoto T, Nose A. A group of segmental premotor interneurons regulates the speed of axial locomotion in Drosophila larvae. Curr Biol. 2014;24(22):2632-42. https://doi.org/10.1016/j.cub.2014.09.026.

65. Zwart MF, Pulver SR, Truman JW, Fushiki A, Fetter RD, Cardona A, et al. Selective inhibition mediates the sequential recruitment of motor pools. Neuron. 2016;91(3):615-28. https://doi.org/10.1016/j.neuron.2016.06.031.

66. Kohsaka H, Guertin PA, Nose A. Neural circuits underlying fly larval locomotion. Curr Pharm Des. 2017;23(12):1722-33. https://doi.org/10.21 74/1381612822666161208120835

67. Clark MQ, Zarin AA, Carreira-Rosario A, Doe CQ. Neural circuits driving larval locomotion in Drosophila. Neural Dev. 2018;13:1-10.

68. Berni J. Genetic dissection of a regionally differentiated network for exploratory behavior in drosophila larvae. Curr Biol. 2015;25(10):1319-26. https://doi.org/10.1016/j.cub.2015.03.023.

69. Ainsley J, Pettus J, Bosenko D, Gerstein C, Zinkevich N, Anderson M, et al. Enhanced locomotion caused by loss of the Drosophila DEG/ENaC protein Pickpocket1. Curr Biol. 2003;13(17):1557-63. https://doi.org/10.1016/509609822(03)00596-7.

70. Heckscher ES, Lockery SR, Doe CQ. Characterization of Drosophila larval crawling at the level of organism, segment, and somatic body wall musculature. J Neurosci. 2012;32(36):12460-71. https://doi.org/10.1523/ JNEUROSCI.0222-12.2012.

71. Lahiri S, Shen K, Klein M, Tang A, Kane E, Gershow M, et al. Two alternating motor programs drive navigation in Drosophila larva. PLoS One. 2011;6(8): e23180. https://doi.org/10.1371/journal.pone.0023180.

72. Hernandez-Nunez L, Belina J, Klein M, Si G, Claus L, Carlson JR, et al. Reverse-correlation analysis of navigation dynamics in Drosophila larva using optogenetics. Elife. 2015;4:1-16.

73. Gepner R, Skanata MM, Bernat NM, Kaplow M, Gershow M. Computations underlying Drosophila photo-taxis, odor-taxis, and multi-sensory integration. Elife. 2015:1-21.

74. Pehlevan C, Paoletti P, Mahadevan L. Integrative neuromechanics of crawling in D. Melanogaster Larvae. Elife. 2016;5:1-23.

75. Loveless J, Webb B. A neuromechanical model of larval chemotaxis. Integr Comp Biol. 2018:58:906-14.

76. Loveless J, Lagogiannis K, Webb B. Modelling the neuromechanics of exploration and taxis in larval Drosophila. PLoS Comput Biol. 2019:1-33.

77. Gjorgjieva J, Berni J, Evers JF, Eglen SJ. Neural circuits for peristaltic wave propagation in crawling Drosophila larvae: analysis and modeling. Front Comput Neurosci. 2013;7:1-19.

78. Sun X, Liu Y, Liu C, Mayumi K, Ito K, Nose A, et al. A neuromechanical model and kinematic analyses for Drosophila larval crawling based on physical measurements. bioRxiv. 2020.

79. Dillon ME, Wang G, Garrity PA, Huey RB. Thermal preference in Drosophila. J Therm Biol. 2009;34(3):109-19. https://doi.org/10.1016/j.jtherbio.2008.11.007.

80. Ni L, Klein M, Svec K V., Budelli G, Chang EC, Ferrer AJ, et al. The ionotropic receptors IR21a and IR25a mediate cool sensing in Drosophila. Elife. 2016;5.

81. Rosenzweig M, Kang KJ, Garrity PA. Distinct TRP channels are required for warm and cool avoidance in Drosophila melanogaster. Proc Natl Acad Sci U S A. 2008;105(38):14668-73. https://doi.org/10.1073/pnas.0805041105.

82. Shen WL, Kwon Y, Adegbola AA, Luo J, Chess A, Montell C. Function of rhodopsin in temperature discrimination in Drosophila. Science. 2011; 331(6022):1333-6. https://doi.org/10.1126/science.1198904.

83. Kwon Y, Shim HS, Wang X, Montell C. Control of thermotactic behavior via coupling of a TRP channel to a phospholipase $\mathrm{C}$ signaling cascade. Nat Neurosci. 2008;11(8):871-3. https://doi.org/10.1038/nn.2170

84. Katoh K, Standley DM. MAFFT multiple sequence alignment software version 7: improvements in performance and usability. Mol Biol Evol. 2013; 30(4):772-80. https://doi.org/10.1093/molbev/mst010.

85. Kohsaka H, Matsuo Y. FIM Track data of larval locomotion of 11 species in the genus Drosophila. figshare https://doi.org/10.6084/m9.figshare.15041829 (2021)

\section{Publisher's Note}

Springer Nature remains neutral with regard to jurisdictional claims in published maps and institutional affiliations. 